\title{
Algebras of Functions on Compact Quantum Groups, Schubert Cells and Quantum Tori
}

\author{
Serge Levendorskiii ${ }^{1}$ and Yan Soibelman ${ }^{2}$ \\ 1 Rostov Institute for National Economy, SU-344798 Rostov-on-Don, USSR \\ 2 Rostov State University, SU-344006, Rostov-on-Don, USSR \\ Received October 23, 1990
}

\begin{abstract}
The structures of Poisson Lie groups on a simple compact group are parametrized by pairs $(a, u)$, where $a \in R, u \in \Lambda^{2} \mathfrak{h}_{R}$, and $\mathfrak{h}_{R}$ is a real Cartan subalgebra of complexification of Lie algebra of the group in question. In the present article the description of the symplectic leaves for all pairs $(a, u)$ is given. Also, the corresponding quantized algebras of functions are constructed and their irreducible representations are described. In the course of investigation Schubert cells and quantum tori appear. At the end of the article the quantum analog of the Weyl group is constructed and some of its applications, among them the formula for the universal $R$-matrix, are given.
\end{abstract}

\section{Introduction}

0.1 . Let $G$ be a finite-dimensional simply connected simple complex Lie group. $G$ is called a Poisson Lie group ([3]) if it is a Poisson manifold and the multiplication $\mu: G \times G \rightarrow G$ is a morphism of Poisson manifolds. There exist many Poisson Lie group structures on the fixed group $G$. All of them are listed in [1].

Let $K \subset G$ be a maximal compact subgroup. The results of [1] imply (see $[28,29]$ and Sect. 1 below) that all Poisson Lie group structures on $K$ are parameterized essentially by pairs $(a, u)$, where $a$ is a real number, $u \in \Lambda^{2} \mathfrak{h}_{\mathbb{R}}$ and $\mathfrak{h}_{\mathbb{R}}$ is a real Cartan subalgebra of the Lie algebra $g=\operatorname{Lie} G$.

Fix pair $(a, u)$. The corresponding Poisson Lie group is denoted by $K(a, u)$. In Drinfeld's theory [3], the group $K(a, u)$ may be viewed as a classical object that is subject to quantization. To formulate this in the language of algebras of functions, consider the algebra of regular functions $\mathbb{C}[G]$ on a Poisson Lie group $G$. This is a Poisson Hopf algebra in the sense of [3], i.e. the comultiplication $\delta: \mathbb{C}[G]$ $\rightarrow \mathbb{C}[G] \otimes \mathbb{C}[G]$ is a Poisson algebra homomorphism. Consider the algebra of $K$-finite functions $\mathbb{C}[K]$ consisting of restrictions $\left.f\right|_{K}$, where $f \in \mathbb{C}[G]$. The algebra $\mathbb{C}[K]$ has involution (complex conjugation $f \mapsto \bar{f}$ ), and the comultiplication $\delta: \mathbb{C}[K] \rightarrow \mathbb{C}[K] \otimes \mathbb{C}[K]$ is a homomorphism of algebras with involution 
As a Hopf algebra with involution, $\mathbb{C}[K]$ is isomorphic to $(\mathbb{C}[G], *)$, where $f^{*}(g)$ $=\overline{f\left(\omega_{0}(g)\right)}$. Here $\omega_{0}: G \rightarrow G$ is the Cartan anti-involution such that $K$ is the set of fixed points of $\omega_{0}$.

The result of quantization of $K(a, u)$ is the family $\mathbb{C}[K(a, u)]_{q}$ of Hopf algebras with involution, where $q \geqq 1$ is quantization parameter and $\mathbb{C}[K(a, u)]_{1}$ $=\mathbb{C}[K(a, u)]$. One constructs this family in two steps. First, one quantizes the Poisson Hopf algebra $\mathbb{C}[G(a, u)]$ as indicated in [3]. The result is the family $\mathbb{C}[G(a, u)]_{q}$ of Hopf algebras, where $q \in \mathbb{C} \backslash 0$ (and $q$ isn't a root of unity).

To make the second step, recall that $\mathbb{C}[G(a, u)]_{q}$ is a Hopf algebra consisting of matrix elements of finite-dimensional representations of the quantized universal enveloping algebra $U_{q}(g)$. It follows that in $\mathbb{C}[G(a, u)]_{q}, q \in \mathbb{R}$, one can introduce an involution *. By the Hopf algebra $\mathbb{C}[K(a, u)]_{q}$, we mean the pair $\left(\mathbb{C}[G(a, u)]_{q}, *\right)$. We also call it the algebra of $K$-finite functions on quantum group $K(a, u)$ or the quantized algebra of $K$-finite functions on group $K(a, u)$.

The cases $q \geqq 1$ and $q \leqq 1$ being analogous, we may and shall assume that $q \geqq 1$.

0.2 . In this paper we'll deal with irreducible $*$-representations of the algebra $\mathbb{C}[K(a, u)]_{q}$ in a Hilbert space, by bounded operators. In the case $q=1$ the description of such representations is trivial: all irreducible representations are one-dimensional and correspond to points of group $K$.

In the case $q \neq 1$ the situation changes drastically: there exist infinitedimensional irreducible representations of the algebra $\mathbb{C}[K(a, u)]_{q}$. Let $\pi$ be one of these representations; then $\pi$ determines two-sided $*$-ideal $I_{\pi}=\operatorname{Ker} \pi \subset \mathbb{C}[K(a, u)]_{q}$. As $q \rightarrow 1$, the ideal $I_{\pi}$ "tends" to a Poisson *-ideal $\bar{I}_{\pi} \subset \mathbb{C}[K(a, u)]$. $\pi$ being irreducible, $\bar{I}_{\pi}$ is a maximal Poisson $*$-ideal. Hence, there is a minimal Poisson submanifolds in $K(a, u)$ (i.e. symplectic leaf) corresponding to $\bar{I}_{\pi}$. We see that representations of $\mathbb{C}[K(a, u)]_{\text {q }}$ are closely related to symplectic leaves of the Poisson structure in $K(a, u)$. The picture of these leaves depends on parameters $(a, u)$. In the case $a=0, u=0$ the symplectic leaves are points, $\mathbb{C}[K(0,0)]_{q}=\mathbb{C}[K]$, and all (irreducible $*$-) representations are one-dimensional. In the case $a \neq 0, u=0$, symplectic leaves are naturally isomorphic to Schubert cells of the full flag manifold $K / T$, where $T$ is the maximal torus. As it turns out (see [28, 29] and Sect. 3 below), the geometry of Schubert cells and that of flag variety govern the representation theory of the corresponding quantized algebra of functions. One can develop this relation further. For one possible direction see [14] and Sect. 5 below. This is a quantum analog of the Weyl group. Here is an illustration for the typical case $a=1$. For the sake of brevity, denote $\mathbb{C}[K(1,0)]_{q}$ by $\mathbb{C}[K]_{q}$ and note that for every element $w$ of the Weyl group $W$ there exists an irreducible representation $\pi_{w}$ of algebra $\mathbb{C}[K]_{q}$ which is in a sense a representation with the highest vector $e_{w}$ (see [29] and Sect. 3 below). This allows to define the functional on the algebra $\mathbb{C}[K]_{q}$ as follows: $\bar{w}(f)=\left(\pi_{w}(f) e_{w}, e_{w}\right)$. It turns out that if $\left\{s_{i}\right\}_{i=1}^{m}$ are simple reflections then for $\left\{\bar{s}_{i}\right\}_{i=1}^{m}$ the Coxeter relations hold. This is due to the fact that if $w=s_{1_{1}} \ldots s_{i_{k}}$ is a reduced expression, then $\pi_{w} \cong \pi_{s_{t_{1}}} \otimes \ldots \otimes \pi_{s_{i_{k}}}$, where the right side is independent of the choice of reduced expression. The relation $\bar{s}_{i}^{2}=1$ doesn't hold; nevertheless, one manages to construct Hopf algebra containing all $\bar{s}_{i}$. In contrast to the classical case $q=1$, this algebra is infinite-dimensional. It is the quantum analog of the minimal Hopf algebra containing the universal enveloping algebra $U(g)$ and simple reflections $\left\{s_{i}\right\}_{i=1}^{m}$.

Let's go back to a description of irreducible representations of the algebra $\mathbb{C}[K(a, u)]_{q}$. As it was noted, in the case $a \neq 0, u=0$ these representations are described in terms of geometry of Schubert cells and that of flag variety. In the case 
$a \neq 0, u \neq 0$ we have essentially a different picture. Here the symplectic leaves are a "perturbation in parameter $u$ " of the Schubert cells. There appear everywhere dense symplectic leaves and the space of these leaves becomes non-semiseparable with the natural topology. In the representation theory of the algebra $\mathbb{C}[K(a, u)]_{q}$ the main examples for non-commutative geometry by Connes, namely the quantum tori, appear (for references on the latter see, for example, [23]). We believe this is of interest because quantum tori aren't quantum groups.

In the last case $a=0, u \neq 0$ Schubert cells do not play any role but quantum tori still do.

0.3 . Recall briefly the history of the subject in question. The first results were announced by the second author and Vaksman at the Winter Voronezh Mathematical School (January, 1985; unpublished). They had described all irreducible representations of the algebra $\mathbb{C}[S U(3)]_{q}$. Later this result was generalized by the second author in $[28]$ for $\mathbb{C}[S U(n)]_{q}$ and in $[29]$ for $\mathbb{C}[K]_{q}$. In these articles the relations with Schubert cells and geometry of the flag variety were also established. The case $S U(n)$ (but without relations to geometry) was independently considered in [10]. The case $K(1, u)$ and relations to quantum tori were studied by the first author in [13].

As the first published results on representations of quantum algebras of functions, one should view preprints [26, 27] (part of the results were published in [31]) and article [37]. All these articles dealt with $\mathbb{C}[S U(2)]_{q}$. They contain much more than just a description of representations. For example, in $[26,27,31]$ the representation theory of $\mathbb{C}[S U(2)]_{q}$ was used to construct the harmonic analysis on the quantum group $S U(2)$. One of the consequences of this theory is the relation between quantum groups and the theory of $q$-special functions (this relation was discovered independently in $[21,11])$. For the later developments in this direction, see the review [12].

Also, in [37] the elements of harmonic analysis and differential calculus on the quantum group $S U(2)$ were constructed. Later, in [38] the axiomatric approach for compact quantum groups was developed. It should be noted that [38] didn't contain the algorithm for quantization of an arbitrary simple compact Lie group. This algorithm (described in Subsect. 0.1) follows from results [3]. There exists another method of quantization, based on the notion of the quantum $R$-matrix (see [5]). The methods of [38] and [5] are more algebraic-geometric than that of [3], since the former start from a coordinate ring of an algebraic variety. The approach [3] is similar to that of the representation theory and differential geometry (one starts from an infinitesimal object, namely, the quantum universal enveloping algebra). A more systematic "algebraic-geometric" approach may be found in [20]: coordinate rings of quantum linear spaces are initial objects and coordinate rings of quantum groups appear as Hopf algebras of their (co-)endomorphisms.

In the abovementioned $[5,20,38]$ the authors do not investigate the representations of quantum algebras of functions, but in these articles useful information about the structure of these algebras may be found.

We do not mention here the articles on quantum algebras of functions on noncompact groups since they have no immediate connections with the present article.

0.4. In the present article we give a full description of irreducible representations of algebras of functions on compact quantum groups, the latter being supposed to arise as the results of quantization of Poisson structures from the list in [1]. Our 
description is based essentially on $[13,29]$ and we reproduce part of their results here, one of the reasons being the misprints in [29] (there were none of them in the preprint).

0.5. In Sect. 1 of the present article the "classical theory" is described, i.e. compact Poisson Lie groups, Schubert cells and symplectic leaves. In Sect. 2 the quantized universal enveloping algebras and the corresponding quantized algebras of functions are introduced. The representation theory of quantized algebras of functions for the case $a \neq 0, u=0$ (i.e. when leaves are isomorphic to Schubert cells) is developed in Sect. 3. The generalization of results of Sect. 3 is given in Sect. 4, where quantum tori appear. In the case $a \neq 0, u \neq 0$ the representation theory is the "semidirect product" of the results of Sect. 3 and of the representation theory of quantum tori.

At the end of Sect. 4 the last case $a=0, u \neq 0$ is considered. Here the same ideas, as in the case $a \neq 0, u \neq 0$, allow to reduce the representation theory to one of quantum tori and one of some factor-algebras of (classical) algebras of functions on group $K$. Note that this case can be put into the framework of deformation quantization by Rieffel (see, for example, [23]). Also, in this case one can attempt to state and solve all the problems of the theory of quantum tori (see [23] for a review on the latter).

An algebra of functions on a compact quantum group admits $C^{*}$-completion. In the case of Sect. 3 (i.e. for $a \neq 0, u=0$ ) we obtain an algebra of type I, but in the case of Sect. $4(a \neq 0, u \neq 0)$ we obtain algebras of other type for almost all $u$ and $q$. One can see that on the "quasi-classical level" already the general case of Sect. 4 corresponds to irrational rotations of torus and to non-semiseparable spaces of symplectic leaves. This indicates the relations to the theory of wild Lie groups.

In Sect. 5, the definition of quantum Weyl group [14] and some applications are given, all of them being related to the case of Sect. 3 only, i.e. to the case of the standard Poisson structure on a compact group. We omit proofs given in [14], but sometimes we give proofs of the results that were only stated in [14].

At the beginning of each section we give a brief abstract of the results it contains.

0.6. The present article isn't a review of the representation theory of quantum algebras of functions and the bibliography is not exhaustive. Some of the results were discovered several times and we refer to the earliest articles we know of.

0.7. Notations. The letter $G$ denotes a simple complex Lie group, $g=\operatorname{Lie} G$ is its Lie algebra. We fix a triangular decomposition $g=n_{-} \oplus \mathfrak{h} \oplus n_{+}$, where $n_{ \pm}$are nilpotent subalgebras and $\mathfrak{h}$ is a Cartan subalgebra. Let $\Delta=\Delta_{+} \cup \Delta_{-}$be the corresponding decomposition of the root system. We fix a basis $\left\{\alpha_{i}\right\}_{i=1}^{m}$ in $\Delta_{+}$, an invariant inner product (, ) on $\mathfrak{h}^{*}$, Cartan-Weyl basis $\left\{X_{\alpha}\right\}_{\alpha \in \Delta} \cup\left\{H_{i}\right\}_{i=1}^{m}$ in $g$ such that $H_{i}$ corresponds to $\alpha_{i}$ under isomorphism $\mathfrak{h}^{*} \cong \mathfrak{h}$.

Let $\omega_{0}: X_{\alpha_{i}} \mapsto-X_{-\alpha_{i}}$ be an antilinear Cartan involution. It defines the real Lie subalgebra $k \subset g$, the real Cartan subalgebra $\mathfrak{h}_{\mathbb{R}}=\bigoplus_{i=1}^{m} \mathbb{R} H_{i}$ and the maximal compact subgroup $K \subset G$. The letter $W$ denotes the Weyl group of $K$ and $T$ denotes the maximal torus in $K . U(g)$ denotes the universal enveloping algebra.

The set of dominant (of regular dominant) weights of $g$ is denoted by $P_{+}\left(P_{++}\right)$. The set of weights of a simple $g$-module $L(\Lambda)$ with the highest weight $\Lambda$ is denoted by $P(\Lambda)$. 


\section{Compact Poisson Groups}

1.1. In this section we give the description of all structures of Poisson Lie groups on a simple compact Lie group. They are parameterized by pairs $(a, u)$ with $a \in \mathbb{R}$ and $u \in \Lambda^{2} \mathfrak{h}_{\mathbb{R}}$. For all pairs $(a, u)$ the symplectic leaves are described. The pictures differ significantly in cases: 1) $a=0, u=0$; 2) $a \neq 0, u=0$; 3) $a \neq 0, u \neq 0$; 4) $a=0$, $u \neq 0$. There is also the dependence on $u \neq 0$, but "for almost all" $u \neq 0$ the picture is essentially the same (and depends on whether $a=0$ or not).

1.2. Let $\mathbb{C}[G]$ denote the algebra of regular functions on group $G$. It is well-known that $\mathbb{C}[G]$ consists of matrix elements $\ell(\varrho(g) v)$ of finite-dimensional analytic representations $\varrho: G \rightarrow$ End $V$. Let

$$
r=\frac{i}{2} \sum_{\alpha \in \Delta_{+}}\left(X_{-\alpha} \otimes X_{\alpha}-X_{\alpha} \otimes X_{-\alpha}\right) \in \Lambda^{2} g .
$$

Fix $a \in \mathbb{R}, u \in \Lambda^{2} \mathfrak{h}_{\mathbb{R}}$ and define on $G$ a Poisson structure as follows:

$$
\begin{aligned}
& \left\{\ell_{1}\left(\varrho_{1}(g) v_{1}\right), \ell_{2}\left(\varrho_{2}(g) v_{2}\right)\right\} \\
& \quad=\left(\ell_{1} \otimes \ell_{2}\right)\left(\left[\left(\varrho_{1} \otimes \varrho_{2}\right)(a r+u), \varrho_{1}(g) \otimes \varrho_{2}(g)\right] v_{1} \otimes v_{2}\right) .
\end{aligned}
$$

In (1.1), $\ell_{i}\left(\varrho_{i}(g) V_{i}\right)$ are matrix elements and $[$,$] is the commutator of linear$ operators.

Definition [3]. Let $G$ be a Lie group equipped with a Poisson structure. $G$ is called a Poisson Lie group if the multiplication map $\mu: G \times G \rightarrow G$ is a Poisson map, $G \times G$ being equipped with the product Poisson structure.

1.2.1. Proposition $[28,29]$. a) Formula (1.1) equips $G$ with a Poisson Lie group structure.

b) The restriction of bracket (1.1) on $K$ makes $K$ a Poisson Lie group.

c) For every Poisson Lie group structure on $K$ there exist $a \in \mathbb{R}, u \in \Lambda^{2} \mathfrak{h}_{\mathbb{R}}$ such that the corresponding Poisson bracket can be reduced to form (1.1) by means of an inner automorphism of $K$ and multiplication of bracket by complex number.

Proof. a) is well-known and b) follows from a). c) Sects. 6 and 8 of [1] imply that a general form of a real (i.e. $\omega_{0}$-invariant) solution of a modified Yang-Baxter equation is just $a r+u$. Each such solution defines a Poisson Lie group structure on $K$ and it remains to note that one can multiply a Poisson bracket by complex number and get a Poisson Lie group structure.

The Poisson Lie groups corresponding to $(a, u)$ will be denoted by $G(a, u)$ and $K(a, u)$.

1.3. In this subsection, we'll describe symplectic leaves [35] corresponding to Poisson structures from Proposition 1.2.1 with $a \neq 0, u=0$. We can assume that a Poisson structure on $K$ is defined by the Yang-Baxter tensor

$$
r_{0}=\frac{1}{2} \sum_{\alpha \in \Delta_{+}}\left(X_{-\alpha} \otimes X_{\alpha}-X_{\alpha} \otimes X_{-\alpha}\right)
$$

then (cf. (1.1)). For the sake of brevity we use here notations $G, K$ for the corresponding Poisson Lie groups $G(1,0), K(1,0)$.

For an element $w$ of Weyl group $W$ choose its representative in $K$ as in [34]. This representative will be denoted by $w$, too. Finally, let $K=\bigcup_{w \in W} K_{w}$ be a Bruhat
decomposition [34]. 
1.3.1. Proposition $[28,29,36]$. Let $a \neq 0, u=0$. Then a) every symplectic leaf lies in its entirety in some Bruhat class $K_{w}$, and

b) every symplectic leaf $\Sigma \subset K_{w}$ is of the form $\Sigma_{w} \cdot t$ (or $t_{1} \cdot \Sigma_{w}$ ).

Here $\Sigma_{w}$ is standard symplectic leaf through $w$, and $t, t_{1} \in T$, where $T$ is the maximal torus in $K$.

Proof. Symplectic leaves may be described by means of [24] provided "the classical double" for $K[3]$ is constructed. One sees easily that this double is just the group $G$, provided the dual group to $K$ in the sense of Drinfeld is identified with subgroup $A N$ in the Iwasawa decomposition $G=K A N$. This follows from decomposition $g=k \otimes$ Lie $A N$ since the summands $k$ and Lie $A N$ are isotropic with respect to the imaginary part of the Killing form.

1.2.3. Let $i \in[1, m]$ be a vertex of Dynkin diagram of Lie algebra $g$ and let $\gamma_{i}: S U(2) \rightarrow K$ be the canonical Poisson Lie group embedding corresponding to this vertex. Recall that

$$
\mathscr{D}=\left\{\left(\begin{array}{c}
a-b \\
b \bar{a}
\end{array}\right) \in S U(2) \mid b<0\right\}
$$

is a symplectic leaf in $S U(2)$ [31] and set $\Sigma_{i}=\gamma_{i}(\mathscr{D})$.

1.3.3. Proposition. a) $\Sigma_{i}$ is a symplectic leaf in $K$.

b) If $w=s_{i_{1}} \ldots s_{i_{k}}$ is a reduced expression in Weyl group, then $\Sigma_{i_{1}} \ldots \Sigma_{i_{k}}$ is a symplectic leaf in $K$ which coincides with the leaf $\Sigma_{w}$ from Proposition 1.3.1b).

Proof follows from the definition of a Poisson Lie group since $\gamma_{i}$ is a Poisson embedding.

1.3.4. Remark. Note that the multiplication by an element of maximal torus $T$ from the left or from the right is a Poisson map. Hence, on the flag manifold $K / T$ the Poisson structure such that projection $\pi: K \rightarrow K / T$ is a Poisson map is well defined.

1.3.5. Corollary. a) Every symplectic leaf in $K$ is isomorphic to a product of twodimensional leaves or coincides with the point of $T$.

b) Let $K / T=\bigcup_{w \in W} X_{w}$ be a Schubert cell decomposition [24]. Then $X_{w}=\pi\left(\Sigma_{w}\right)$ is a symplectic leaf and every symplectic leaf in $K / T$ is of this form.

1.3.6. Remark. The decomposition of $K / T$ in Schubert cells is the consequence of Bruhat decomposition. The "symplectic" point of view on Bruhat decomposition is given by Corollary 1.3.5b): the Poisson manifold $K / T$ is the union of symplectic leaves.

1.4. Here we consider the case $a \neq 0, u \neq 0$. We may assume that the Poisson structure is determined by tensor $r_{0}+i u$; the corresponding Poisson Lie group $K(1, u)$ will be denoted by $K^{u}$ in this subsection.

The Killing form $Q$ in $g$ is defined by conditions

$$
Q\left(X_{\alpha}, X_{-\alpha}\right)=1, \quad Q\left(H_{i}, H_{i}\right)=\left(\alpha_{i}, \alpha_{i}\right), \quad Q\left(X_{\alpha}, H_{i}\right)=0 .
$$

Let $\hat{u}: \mathfrak{h}_{\mathbb{R}}^{*} \rightarrow \mathfrak{h}_{\mathbb{R}}$ be the operator corresponding to tensor $u \in \Lambda^{2} \mathfrak{h}_{\mathbb{R}}$ under isomorphism $\mathfrak{h}_{\mathbb{R}} \cong \mathfrak{h}_{\mathbb{R}}^{*}$ defined by the Killing form. Set

$$
\mathfrak{h}_{\mathbb{R}}^{u}=(I+2 i \hat{u}) \mathfrak{h}_{\mathbb{R}}, \quad g_{2}^{u}=\mathfrak{h}_{\mathbb{R}}^{u} \oplus n_{+}, \quad g_{1}=k=\operatorname{Lie} K .
$$


1.4.1. Proposition. The triple $\left(g, g_{1}, g_{2}^{u}\right)$ is a Manin triple in the sense of [1].

Proof is analogous to that of Proposition 1.3.1: $g_{1}$ and $g_{2}^{u}$ are both isotropic with respect to imaginary part of the Killing form.

1.4.2. Let $B^{u}$ be a subgroup in $G$ corresponding to Lie algebra $g_{2}^{u}$. We have $G=K B^{u}$. Note that $B^{0}=A N$.

1.4.3. Proposition [13]. a) Every symplectic leaf $\Sigma^{u}$ of the Poisson Lie group $K^{u}$ lies in some Bruhat class $K_{w}$.

b) If $\Sigma^{u} C K_{w}$ is a leaf through a point $t \cdot w$, where $t \in T$, then $\Sigma^{u}=T_{w} \cdot t \cdot \Sigma_{w}$, where $\Sigma_{w}$ is the standard symplectic leaf from Proposition 1.3.1.b), $T_{w}=\exp \left(i\left(\tilde{u}-w^{-1} \hat{u} w\right) \mathfrak{h}_{\mathbb{R}}\right)$ is a subgroup in $T$ and $\hat{u}: \mathfrak{h}_{\mathbb{R}} \rightarrow \mathfrak{h}_{\mathbb{R}}$ is the operator corresponding to tensor $u \in \Lambda^{2} \mathfrak{h}_{\mathbb{R}}$.

Proof. A general form of element of the group $B^{u}$ is $x_{+} \exp ((I+2 i \hat{u}) \ell)$ where $x_{+} \in N_{+}, \ell \in \mathfrak{h}_{\mathbb{R}}^{*}$. It follows from [24] that the symplectic leaf through $y$ is of the form $\Sigma^{u}(y)=B^{u} y B^{u} \cap K$, i.e.

$$
\Sigma^{u}(y)=\left\{z \in K \mid \exists p, q \in B^{u}: p y=z q\right\} .
$$

This and equality $N_{+} w=\Sigma_{w} N_{+}$give $\Sigma^{u}(\mathrm{y})=\mathrm{T}_{w} \cdot \mathrm{t} \cdot \Sigma_{w}$ and to conclude the proof we note that Poisson manifolds $K, K^{u}$ are disjoint unions of their symplectic leaves.

1.5. Now we consider the last case $a=0, u \neq 0$. The corresponding Poisson Lie group is denoted by $K(0, u)$. The "classical double" for group $K(0,0)$ is $T^{*} K$ $=K \times k^{*}$ with multiplication $\left(g_{1}, \lambda_{1}\right) \cdot\left(g_{2}, \lambda_{2}\right)=\left(g_{1} g_{2}, \lambda_{2}+\operatorname{Ad}_{g_{2}{ }_{1}}^{*} \lambda_{1}\right)$ and the "classical double" for the corresponding bialgebra Lie [3] $k$ is $\mathscr{D}(k)=k \oplus k^{*}$, where the bracket on $k$ is initial, the bracket on $k^{*}$ is trivial and the bracket on $\mathscr{D}(k)$ is defined by

$$
[x+\xi, y+\eta]=[x, y]+\operatorname{ad}_{x}^{*} \eta-\operatorname{ad}_{y}^{*} \xi
$$

for all $x, y \in k, \xi, \eta \in k^{*}$.

These results give the following propositions:

1.5.1. Proposition. a) The "classical double" for the Poisson Lie group $K(0, u)$ is $T^{*} K=K \times k^{*}$ with the multiplication law introduced above.

b) The group dual to $K(0, u)$ in the same sense of Drinfeld is a subgroup in $T^{*} K$ with Lie algebra $(I+\hat{u})\left(i \mathfrak{h}_{\mathbb{R}}^{*}\right)^{*} \oplus v^{*}$, where $k=i \mathfrak{h}_{\mathbb{R}} \oplus v$ is an orthogonal decomposition with respect to Killing form and $\left(i \mathfrak{h}_{\mathbb{R}}\right)^{*}, v^{*}$ are dual subspaces in $k^{*}$.

Proof. It suffices to note that $\left(\mathscr{D}(k), k,(I+\hat{u})\left(i \mathfrak{h}_{\mathbb{R}}\right)^{*} \oplus v^{*}\right)$ is a Manin triple, provided the invariant bilinear form in $\mathscr{D}(k)$ is given by the usual formula

$$
\langle x+\xi, y+\eta\rangle=\langle x, \eta\rangle+\langle y, \xi\rangle, \quad x, y \in k, \quad \xi, \eta \in k^{*} .
$$

1.5.2. Proposition. Symplectic leaf through a point $g$ in $K(0, u)$ is of the form

$$
\Sigma_{g}^{0, u}=\left\{\exp (\hat{u} \lambda) g \exp \left(-\hat{u} p r_{1} \operatorname{Ad}_{g-1}^{*}(\lambda, \xi)\right) \mid \lambda \in\left(i \mathfrak{h}_{R}\right)^{*}, \xi \in v^{*}\right\},
$$

where $\mathrm{pr}_{1}:\left(i \mathfrak{h}_{\mathbb{R}}\right)^{*} \oplus v^{*} \rightarrow\left(i \mathfrak{h}_{\mathbb{R}}\right)^{*}$ is a projection.

Proof is analogous to that of Proposition 1.4.3.

1.6. Remark. In the next sections we'll obtain the quantum counterparts of results of Subsect. 1.3 in the spirit of the Kirillov-Kostant method of orbits: we'll attach to each symplectic leaf an (irreducible) representation of the quantum algebra of 
functions. This correspondence can fail for some $u \neq 0$ (i.e. in the cases considered in 1.5, 1.4). In addition, for almost all $u \neq 0$ the space of symplectic leaves is nonsemiseparable and these two facts are closely related. Really, it is well-known [24] that symplectic leaf through the point $g \in K$ coincides with the orbit through $g$ of the right (or left) dressing action by the dual Poisson Lie group in the sense of Drinfeld. If $K$ is equipped with the trivial bracket then the dual group is coalgebra $k^{*}$ equipped with a Lie-Berezin-Kirillov bracket and the dressing action is the usual coadjoint action. Non-semiseparableness of the space of orbits indicates that $K$ is a wild Lie group [7]. For such a group the correspondence "leaf $\leftrightarrow$ representation" can fail. We encounter with the same effect here.

\section{Quantized Universal Enveloping Algebras and Quantized Algebras of Functions}

2.1. At the beginning of the section we recall some basic definitions related to Hopf *-algebras. After these we give the examples, namely, quantized universal enveloping algebras and quantized algebras of functions.

2.2. Let $F$ be a commutative unital ring and let $\lambda \mapsto \bar{\lambda}$ be an involutive automorphism of $F$. We consider quantum groups $A$ over $F$. This means [3] that $A$ is a Hopf algebra over $F$ with invertible antipode $S: A \rightarrow A$.

2.2.1. Definition $[5,26,28,29,38]$. $A$ is called a Hopf $*$-algebra if it is equipped with an involution $a \mapsto a^{*}$ such that for all $a, b \in A, \lambda \in F$ :

a) $(\lambda a)^{*}=\bar{\lambda} a^{*}$,

b) $(a b)^{*}=b^{*} a^{*}$,

c) $\Delta\left(a^{*}\right)=\Delta(a)^{*}$.

Here $(a \otimes b)^{*}=a^{*} \otimes b^{*}$.

Note that a)-c) imply $\left(S\left(S(a)^{*}\right)\right)^{*}=a$ (cf. [38]).

For Hopf *-algebra $A$ the dual Hopf algebra $A^{V}=\operatorname{Hom}_{F}(A, F)$ is a Hopf *-algebra as well, the involution being defined by

for all $a \in A$.

$$
\ell^{V}(a)=\overline{\ell\left(S(a)^{*}\right)}
$$

2.3. Let $C_{A}$ be a category of $A$-modules which are free over $F$ and have finite rank over $F$. One defines associative tensor multiplication in $C_{A}$ : for $M_{1}, M_{2} \in \mathrm{Ob} C_{A}$ the $F$-module $M_{1} \underset{F}{\otimes} M_{2}$ is equipped with $A$-module structure by formula

$$
a \cdot\left(m_{1} \otimes m_{2}\right)=\Delta(a) \cdot\left(m_{1} \otimes m_{2}\right),
$$

where $(x \otimes y)\left(m_{1} \otimes m_{2}\right)=x m_{1} \otimes y m_{2}$. Furthermore, for every $A$-module $M$ one defines its left dual $M^{V}=\operatorname{Hom}_{F}(M, F)$ by formula

$$
(a \cdot \ell)(b)=\ell(S(a) b)
$$

for every $\ell \in M^{V}, a, b \in A$.

Let $B$ be the set of matrix elements of objects from $C_{A}$, i.e. $B$ consists of pairs $(\ell, m)$, where $m \in M, M \in \mathrm{Ob}_{A}, \ell \in M^{V}$. Then $B$ can be equipped with Hopf algebra structure with invertible antipode. Note that the multiplication is given by formula

$$
\left(\ell_{1}, m_{1}\right) \cdot\left(\ell_{2}, m_{2}\right)=\left(\ell_{1} \otimes \ell_{2}, m_{1} \otimes m_{2}\right),
$$

the $A$-module structure in $M_{1} \otimes M_{2}$ being defined by (2.2).

Clearly, $B$ is a Hopf subalgebra in $A^{V}$. 
2.3.1. Definition. Let $A$ be Hopf $*$-algebra over $\mathbb{C}$ and $M \in \mathrm{Ob} C_{A} . M$ is called a unitarizable module provided it can be equipped with sesquilinear inner product $\langle$,$\rangle such that$

$$
\left\langle a m_{1}, m_{2}\right\rangle=\left\langle m_{1}, a^{*} m_{2}\right\rangle .
$$

Let $\bar{C}_{A}$ be a full subcategory in $C_{A}$, consisting of unitarizable $A$-modules. From (2.1), it follows that $B$ is a Hopf $*$-subalgebra and that the Hopf subalgebra $\bar{B} \subset B$, consisting of matrix elements of objects from $\bar{C}_{A}$ is also a Hopf *-subalgebra.

2.4. Fix $q>1$. Quantized universal enveloping algebra $U_{q}(g)[3,8]$ is a complex Hopf algebra with generators $\left\{X_{i}^{ \pm}, k_{i}^{ \pm}\right\}_{i=1}^{m}$ and defining relations

$$
\begin{gathered}
k_{i}^{ \pm} k_{j}^{ \pm}=k_{j}^{ \pm} k_{i}^{ \pm}, \quad k_{i}^{+} k_{j}^{-}=k_{j}^{-} k_{i}^{+}, \quad k_{i}^{+} k_{i}^{-}=1, \\
k_{i}^{+} X_{j}^{ \pm}=q^{ \pm\left(\alpha_{i}, \alpha_{j}\right) / 2} X_{j}^{ \pm} k_{i}^{+}, \quad k_{i}^{-} X_{j}^{ \pm}=q^{\mp\left(\alpha_{i}, \alpha_{j}\right) / 2} X_{j}^{ \pm} k_{i}^{-}, \\
{\left[X_{i}^{+}, X_{j}^{-}\right]=\delta_{i j} \frac{\left(k_{i}^{+}\right)^{2}-\left(k_{i}^{-}\right)^{2}}{q-q^{-1}},} \\
\sum_{K=0}^{n_{i j}}(-1)^{K}\left(\begin{array}{c}
n_{i j} \\
K
\end{array}\right)_{q_{i}} q_{i}^{-K\left(n_{i j}-K\right) / 2}\left(X_{i}^{ \pm}\right)^{K} X_{j}^{ \pm}\left(X_{i}^{ \pm}\right)^{n_{i j}-K}=0,
\end{gathered}
$$

for $i \neq j$. In formula (2.6), $q_{i}=q^{\left(\alpha_{i}, \alpha_{i}\right) / 2}, n_{i j}=1-a_{i j}$, where $\left(\left(a_{i j}\right)\right)$ is the Cartan matrix of Lie algebra $g$, and

$$
\left(\begin{array}{l}
n \\
K
\end{array}\right)_{t}=\frac{\left(t^{n}-1\right)\left(t^{n-1}-1\right) \ldots\left(t^{n-K+1}-1\right)}{\left(t^{K}-1\right)\left(t^{K-1}-1\right) \ldots(t-1)}
$$

The comultiplication in $U_{q}(g)$ is given by

$$
\Delta\left(X_{i}^{ \pm}\right)=X_{i}^{ \pm} \otimes k_{i}^{+}+k_{i}^{-} \otimes X_{i}^{ \pm}, \quad \Delta\left(k_{i}^{ \pm}\right)=k_{i}^{ \pm} \otimes k_{i}^{ \pm},
$$

and the counit $\varepsilon$ and the antipode $S$ are given by formulae

$$
\begin{gathered}
\varepsilon\left(X_{i}^{ \pm}\right)=0, \quad \varepsilon\left(k_{i}^{ \pm}\right)=1, \\
S\left(X_{i}^{ \pm}\right)=-q_{i}^{ \pm 1} X_{i}^{ \pm}, \quad S\left(k_{i}^{ \pm}\right)=k_{i}^{\mp} .
\end{gathered}
$$

The Hopf algebra $U_{q}(g)$ is equipped with a Hopf $*$-algebra structure by formulae

$$
\left(X_{i}^{ \pm}\right)^{*}=X_{i}^{\mp}, \quad\left(k_{i}^{ \pm}\right)^{*}=k_{i}^{ \pm} .
$$

2.5. Let $F=\mathbb{C} \llbracket h \rrbracket$ be the ring of formal series in $h$. $U_{h}(g)$ denote an $\mathbb{C} \llbracket h \rrbracket$-algebra generated (in the $h$-adic sense, i.e. as an algebra complete in the $h$-adic topology) by generators $\left\{X_{i}^{ \pm}, H_{i}\right\}_{i=1}^{m}$ and defining relations

$$
\begin{gathered}
{\left[H_{i}, H_{j}\right]=0, \quad\left[H_{i}, X_{j}^{ \pm}\right]= \pm\left(\alpha_{i}, \alpha_{j}\right) X_{j}^{ \pm},} \\
{\left[X_{i}^{+}, X_{j}^{-}\right]=\delta_{i j} \frac{\operatorname{sh}\left(\frac{h}{2} H_{i}\right)}{\operatorname{sh} \frac{h}{2}},}
\end{gathered}
$$

the last relation in (2.6) being included. The Hopf *-algebra structure is defined by formulae (2.7)-(2.10) with $k_{i}^{ \pm}=\exp \left( \pm \frac{h}{4} H_{i}\right)$ provided an involution in $\mathbb{C} \llbracket h \rrbracket$ is given by $\lambda h=\bar{\lambda} h$, where $\lambda \in \mathbb{C}$. 
2.5.1. Remark. For Hopf $*$-algebra $U_{h}(g)$, one can repeat all constructions of Subsects. 2.2 and 2.3, considering all modules as free topological modules over $\mathbb{C} \llbracket h \rrbracket$, complete in $h$-topology, and assuming all morphisms to be continuous.

2.5.2. Introduce in $U_{h}(g)$ the new comultiplication by

$$
\Delta_{u}(\xi)=\exp (-i h u / 2) \Delta(\xi) \exp (i h u / 2),
$$

where $\xi \in U_{h}(g)$ and $\Delta$ is given by (2.7). Since $u^{*}=u,(2.11)$ yields $\Delta_{u}\left(\xi^{*}\right)=\Delta_{u}(\xi)$. This means that we've obtained a Hopf *-algebra $U_{h, u}(g)$.

2.6. In this subsection, we set $A=U_{q}(g)$. Consider the full subcategory $\mathscr{D}_{A}$ in $C_{A}$ consisting of objects $M$ such that the spectrum of the morphism $k_{i}^{ \pm}: M \rightarrow M$ consists of positive numbers. It is well-known that $\mathscr{D}_{A} \subset \bar{C}_{A}$ and that every module $M \in \mathrm{Ob} \mathscr{D}_{A}$ is a "quantum deformation" of a finite-dimensional $g$-module $\tilde{M}$ [15]. Using category $\mathscr{D}_{A}$, construct the Hopf algebra $\overline{\mathscr{D}} \subset \bar{B}$ of matrix elements in a way of 2.3 .

2.6.1. Definition. The Hopf algebra $\overline{\mathscr{D}}$ is called the algebra of regular functions on the quantum group $G$, corresponding to fixed $q$, and is denoted by $\mathbb{C}[G]_{q}$.

According to 2.3.1, $\mathbb{C}[G]_{q}$ is a Hopf *-subalgebra in the Hopf *-algebra $U_{q}(g)^{V}$. The involution in $\mathbb{C}[G]_{q}$ is denoted by the same sign *.

2.6.2. Definition. The pair $\left(\mathbb{C}[G]_{q}, *\right)$ is called the algebra of $K$-finite functions on the quantum group $K$, corresponding to fixed $q$ and is denoted by $\mathbb{C}[K]_{q}$.

2.7. In this subsection, we set $A=U_{h, u}(g)$. The category $C_{A}$ consists of topological $A$-modules of finite rank over $\mathbb{C} \llbracket h \rrbracket$ which are free over $\mathbb{C} \llbracket h \rrbracket$ and complete in $h$-adic topology. The corresponding Hopf *-algebra will be denoted by $\mathbb{C}\left[G^{u}\right]_{h}$. It is generated by matrix elements of indecomposable modules from $C_{A}$. It is known [4] that there exists bijection between indecomposable objects from $C_{A}$ and finitedimensional simple $g$-modules. This is the case for the category from 2.6 as well.

Let $L(A)$ be a finite-dimensional simple $U_{q}(g)$-module with the highest weight $\Lambda$. Fix an inner product in $L(\Lambda)$ satisfying (2.6) and choose an orthonormal weight basis $\left\{v_{\lambda}^{(i)}\right\}$ in $L(\Lambda)$ and dual weight basis $\left\{\ell_{-\mu}^{(i)}\right\}$ in $L^{*}(\Lambda)$. Then we can write every matrix element in the form

$$
\ell_{-\mu}^{(j)}\left(\varrho_{\Lambda}(\xi) v_{\lambda}^{(i)}\right)=C_{-\mu, \lambda, \Omega}^{\Lambda}(\xi),
$$

where $\Omega=(j, i) \in \mathbb{N} \times \mathbb{N}, \xi \in U_{q}(g)$ and $\varrho_{A}: U_{q}(g) \rightarrow \operatorname{End} L(\Lambda)$ is the representation corresponding to this element.

We'll use this notation for indecomposable representations of $U_{h, u}(g)$ as well.

If weight space $L(\Lambda)_{\lambda}$ (or $\left.L^{*}(\Lambda)_{-\mu}\right)$ is one-dimensional then we may and shall assume that $\Omega$ is an element of $\mathbb{N} \times 1$ (or $1 \times \mathbb{N}$ ). We shall also use the following notation

$$
\ell_{-\mu}^{(j)}\left(\varrho_{\Lambda}(\xi) v_{\lambda}^{(i)}\right)=C_{-\mu, j, \lambda, i}^{\Lambda}(\xi),
$$

If the weight space is one-dimensional, then the corresponding index is omitted. For example: $C_{-\mu, i, \Lambda}^{\Lambda}, C_{-w A, A}^{\Lambda}$, where $w \in W$ is an element of Weyl group.

From (2.12), we deduce the following formula:

$$
\begin{aligned}
& C_{-\lambda_{1}, \mu_{1}, \Omega_{1}}^{\Lambda_{1_{1}}} \cdot C_{-\lambda_{2}, \mu_{2}, \Omega_{2}}^{\Lambda_{2}} \\
& \quad=\exp \left(\frac{i h}{2}\left(\left(\lambda_{1}, \lambda_{2}\right)_{u}-\left(\mu_{1}, \mu_{2}\right)_{u}\right)\right) C_{-\lambda_{1}, \mu_{1}, \Omega_{1}}^{\Lambda_{1}} \cdot C_{-\lambda_{2}, \mu_{2}, \Omega_{2}}^{\Lambda_{2}} .
\end{aligned}
$$


Here on the right (on the left) stands the multiplication - in $\mathbb{C}\left[G^{u}\right]_{h}$ (the multiplication - in $\mathbb{C}[G]_{q}$, where $\left.q=\exp (h / 2)\right)$ and $(x, y)_{u}=(\check{u} x, y)$, where $\breve{u}: \mathfrak{h}^{*} \rightarrow \mathfrak{h}^{*}$ is the operator corresponding to tensor $u \in \Lambda^{2} \mathfrak{h}_{\mathbb{R}}$ under isomorphism defined by Killing form.

One sees easily that (2.13) is in accordance with the comultiplication $\delta$ in the Hopf algebra $\mathbb{C}\left[G^{u}\right]_{h}$.

2.7.1. Definition. a) The Hopf algebra over $\mathbb{C}$ generated by matrix elements $C_{-\lambda, \mu, \Omega}^{\Lambda} \in \mathbb{C}[G]_{q}, q=\exp (h / 2)$, the multiplication law being defined by (2.13), is called the twisted algebra of regular functions on the quantum group $G^{u}$ (corresponding to parameters $q$ and $u$ ) and denoted by $\mathbb{C}\left[G^{u}\right]_{q}$.

b) Let $*$ be defined by (2.1). The pair $\left(\mathbb{C}\left[G^{u}\right]_{q}, *\right)$ is called the twisted algebra of $K$-finite functions on the quantum group $K$ (corresponding to parameters $q$ and $u$ ) and denoted by $\mathbb{C}\left[K^{u}\right]_{q}$.

2.7.2. Remark. The Hopf algebras $\mathbb{C}[G]_{q}, \mathbb{C}\left[G^{u}\right]_{q}, \mathbb{C}[K]_{q}, \mathbb{C}\left[K^{u}\right]_{q}$ introduced in 2.6.1, 2.6.2, 2.7.1 are the quantizations of Poisson Hopf algebras $[3] \mathbb{C}[G], \mathbb{C}\left[G^{u}\right]$, $\mathbb{C}[K], \mathbb{C}\left[K^{u}\right]$.

The Hopf algebra $\mathbb{C}[G(0, u)]_{q}$ is defined as the algebra generated by matrix elements $C_{-\lambda, \mu, \Omega}^{\Lambda} \in \mathbb{C}[G]$, the multiplication law being given by (2.13) with the multiplication in $\mathbb{C}[G]$ in the right side. In complete analogy with Definition 2.7.1b) we obtain that of the Hopf algebra $\mathbb{C}[K(0, u)]_{q}$. The algebras $\mathbb{C}[G(0, u)]_{q}, \mathbb{C}[K(0, u)]_{q}$ are quantizations of the Poisson Hopf algebras $\mathbb{C}[G(0, u)]$, $\mathbb{C}[K(0, u)]$.

2.7.3. Remark. Considerations of this section show that one may view $\mathbb{C}\left[K^{u}\right]_{q}$ $\left(\mathbb{C}[K(0, u)]_{q}\right)$ as "perturbation in parameter $u \in \Lambda^{2} \mathfrak{h}_{\mathbb{R}}$ " of $\mathbb{C}[K]_{q},(\mathbb{C}[K])$. We'll see below that this principle works in the representation theory of these algebras as well.

2.8. Proposition [29]. In $\mathbb{C}[K]_{q}$, the following equality holds:

$$
\left(C_{-\lambda, \mu, \Omega}^{\Lambda}\right)^{*}=q^{-(\lambda-\mu)(\check{e})} C_{\lambda,-\mu, \Omega}^{-w_{0} \Lambda},
$$

where $w_{0} \in W$ is the longest element in the Weyl group, and $\varrho \in f$ corresponds to the element $\varrho=\frac{1}{2} \sum_{\alpha \in \Delta_{+}} \alpha \in \mathfrak{h}^{*}$ under canonical isomorphism $\mathfrak{h}^{*} \cong \mathfrak{h}$.

Proof. Let $\Omega=(i, j)$. This means $C_{-\lambda, \mu, \Omega}^{\Lambda}(\xi)=\ell_{-\lambda}^{(i)}\left(\varrho_{\Lambda}(\xi) v_{\mu}^{(j)}\right)$, where $\varrho_{A}: U_{q}(g)$ $\rightarrow \operatorname{End} L(\Lambda)$ is the finite-dimensional representation with the highest weight $\Lambda$, $\xi \in U_{q}(g), \ell_{-\lambda}^{(i)} \in L^{*}(\Lambda)_{-\lambda}, v_{\mu}^{(j)} \in L(\Lambda)_{\mu} . L(\Lambda)$ being unitarizable, we can fix the inner product (,) in it such that (2.5) holds. Then we have

$$
\left(C_{-\lambda, \mu, \Omega}^{\Lambda}\right)^{*}(\xi)=\left(\varrho_{\Lambda}(S \xi) v_{\lambda}^{(i)}, v_{\mu}^{(j)}\right)_{L(\Lambda)} .
$$

Note that $U_{q}(g)$-module $L\left(-w_{0} \Lambda\right)$ is isomorphic to $L^{*}(\Lambda)$, the isomorphism being defined by the quantum analog of linear Cartan involution, i.e. by the linear automorphism $\omega: U_{q}(g) \rightarrow U_{q}(g)$ such that

$$
\omega\left(X_{i}^{ \pm}\right)=-q_{i}^{ \pm} X_{i}^{\mp}, \quad \omega\left(k_{i}^{ \pm}\right)=k_{i}^{\mp} .
$$

Set $a=q^{2 \check{\varrho}} \in U_{q}(g)$. It is well-known [4] that $S^{2}(\xi)=a \xi a^{-1}$ for all $\xi \in U_{q}(g)$, where $S$ is the antipode in $U_{q}(g)$. Define the inner product in $L(\Lambda)$ by formula

$$
\left(v_{1}, v_{2}\right)_{L\left(-w_{0} \Lambda\right)}=\left(v_{1}, \varrho_{\Lambda}\left(S^{-1}\left(a^{-1}\right) v_{2}\right)_{L(\Lambda)} .\right.
$$


This is a unitarizing inner product for $\varrho_{\Lambda} \circ \omega$. Further, let $\left\{v_{-\mu}^{(j)}\right\}$ and $\left\{v_{\mu}^{(j)}\right\}$ be orthonormal weight bases in $L\left(-w_{0} \Lambda\right)$ and $L(\Lambda)$, the enumeration in the former being determined by isomorphism $L\left(-w_{0} \Lambda\right) \cong L^{*}(\Lambda)$. Then from (2.14) the desired result follows.

2.8.1. Corollary. Proposition 2.8 holds in $\mathbb{C}\left[K^{u}\right]_{q}$ and in $\mathbb{C}[K(0, u)]_{q}$.

Proof. The formula for involution is independent of $u$.

\section{Irreducible Representations of Quantized Algebra of Functions and Schubert Cells}

3.1. In this section we study irreducible $*$-representations of algebra $\mathbb{C}[G]_{q}$ in a Hilbert space, by bounded operators. We'll call them the irreducible representations of $\mathbb{C}[K]_{q}$. This is consistent with Definition 2.6.2.

First we'll establish the quantum analog of Proposition 1.3.1: each irreducible representation corresponds to the unique symplectic leaf $\Sigma_{w} \cdot t$ (or $t \cdot \Sigma_{w}$ ). The points of maximal torus $T$ correspond to one-dimensional representations and all others are infinite-dimensional representations. If the representation $\tau_{w}$ corresponds to the leaf $\Sigma_{w}$ and $\tau_{t}$ corresponds to the point $t \in T$, then the representation $\pi_{w} \otimes \tau_{t}$ corresponds to the leaf $\Sigma_{w} \cdot t$. Next, we'll establish the quantum analog of Proposition 1.3.3: if $w=s_{i_{1}} \ldots s_{i_{K}}$ is a reduced expression and $\pi_{s_{i_{m}}}$ corresponds to the leaf $\Sigma_{i_{m}}$, then $\pi_{w} \cong \tau_{s_{i_{1}}} \otimes \ldots \otimes \tau_{s_{i_{K}}}$. The proof of this statement is based on a joint article by the second author and Vaksman [31], where the case $K=S U(2)$ (i.e. representations $\pi_{s_{i}}$ ) had been studied. At the end of the section Gelfand-NaimarkSegal states $\bar{w}$ corresponding to representations $\pi_{w}$ are introduced. It is proved that GNS-states $\bar{s}_{i}$ satisfy Coxeter relations. This allows to define the quantum analog of the Weyl group in Sect. 5.

From now on, a representation of $*$-algebra means $*$-representation. We assume all Hilbert spaces to be separable.

3.2. Let $\Lambda \in P_{+}$and let $A_{\Lambda}$ be a subalgebra in $\mathbb{C}[G]_{q}$ generated by matrix elements of the form $C_{-\lambda, \Lambda, \Omega}^{\Lambda}$. Set

$$
A_{+}=\bigoplus_{\Lambda \in \mathbb{P}_{+}} A_{\Lambda}, \quad A_{++}=\underset{\Lambda \in \mathbb{P}_{++}}{\bigoplus} A_{\Lambda}, \quad A_{-}=A_{+}^{*}, \quad A_{--}=A_{++}^{*},
$$

where $X^{*}$ denotes the image of $X$ under involution in $\mathbb{C}[G]_{q}$. The next result can be derived from [22], for example.

3.2.1. Proposition. a) $A$ bilinear map $A_{-} \otimes A_{+} \rightarrow \mathbb{C}[G]_{q}$ is an epimorphism of $\mathbb{C}$-vector spaces.

b) Substitute $A_{++}$for $A_{+}$and $A_{--}$for $A_{-}$; then the statement a) still holds.

One of the main results of this section is the next theorem.

3.3. Theorem. For every irreducible representation $\pi: \mathbb{C}[K]_{q} \rightarrow$ End $H$ in a Hilbert space $H$ there exists $\pi\left(A_{+}\right)$-invariant straight line $\ell \in H$.

3.3.1. Remark. Theorem 3.3 shows that the representation theory of algebra $\mathbb{C}[K]_{q}$ resembles that of algebra $U(g)$ with $A_{+}$in the part of $U\left(b_{+}\right)$. 
We shall divide the proof in two subsections. In the course of the proof we'll establish some results which are interesting per se.

3.4. Fix $\Lambda \in P_{+}, \lambda \in P(\Lambda)$ and consider in $\mathbb{C}[K]_{q}$ the following ideals:

a) $\mathscr{J}_{0}(\lambda, \Lambda)$, namely, the minimal two-sided $*$-ideal containing all matrix elements $C_{-\mu, i, \Lambda}^{\Lambda}$ such that

$$
V_{\mu}^{(i)} \notin U_{q}\left(b_{+}\right)\left(L(\Lambda)_{\lambda}\right) ;
$$

b) $\mathscr{J}_{1}(\lambda, \Lambda)$, namely, the minimal two-sided $*$-ideal containing all matrix elements $C_{-\mu, i, \Lambda}^{\Lambda}$ such that

$$
\mu \geqq \lambda \text { (we write } \mu \geqq \lambda \quad \text { iff } \quad \mu-\lambda=\sum_{i} k_{i} \alpha_{i}, k_{i} \geqq 0 \text { ). }
$$

c) $\mathscr{J}_{2}(\lambda, \Lambda)$, namely, the minimal two-sided $*$-ideal containing all matrix elements $C_{-\mu, i, \Lambda}^{\Lambda}$ such that $\mu<\lambda$.

Clearly, $\mathscr{J}_{2}(\lambda, \Lambda) \subset \mathscr{J}_{1}(\lambda, \Lambda) \subset \mathscr{J}_{0}(\lambda, \Lambda)$. Let $w \in W$. If we set $\lambda=w \Lambda$ in a)-c), then we obtain the ideals which will be denoted by $\mathscr{J}_{0}(w, \Lambda), \mathscr{J}_{1}(w, \Lambda), \mathscr{J}_{2}(w, \Lambda)$.

3.4.1. Proposition. In $\mathbb{C}[K]_{q} / \mathscr{J}_{2}(\lambda, \Lambda)$, the following relations hold:

$$
\begin{aligned}
& C_{-\mu, P, \gamma, q}^{v} \cdot C_{-\lambda, i, \Lambda}^{\Lambda}=q^{-(\Lambda, \gamma)+(\lambda, \mu)} C_{-\lambda, i, \Lambda}^{\Lambda} C_{-\mu, P, \gamma, q}^{v}, \\
& C_{-\mu, P, \Lambda}^{\Lambda} \cdot C_{-\lambda, i, \Lambda}^{\Lambda}=q^{-(\Lambda, \Lambda)+(\lambda, \mu)} C_{-\lambda, i, \Lambda}^{\Lambda} \cdot C_{-\mu, P, \Lambda}^{\Lambda} .
\end{aligned}
$$

Proof. From definition of multiplication in $\mathbb{C}[K]_{q}$ and a general form of universal $R$-matrix of Lie algebra (Sect. 13 of [3]) it follows that

$$
\begin{aligned}
C_{-\mu, P, \gamma, q}^{v} \cdot C_{-\lambda, i, \Lambda}^{\Lambda}= & q^{-(\Lambda, \gamma)+(\lambda, \mu)} C_{-\lambda, i, \Lambda}^{\Lambda} \cdot C_{-\mu, P, \gamma, q}^{v} \\
& +\sum_{\alpha \in \Delta_{+}} \ell_{\alpha}(q) C_{-\lambda+\alpha, i_{\alpha}, \Lambda}^{\Lambda} \cdot C_{-\mu-\alpha, P_{\alpha}, \gamma, q}^{v},
\end{aligned}
$$

where $\ell_{\alpha}(q) \in \mathbb{C}$. To complete the proof it suffices to use the definition of $\mathscr{J}_{2}(\lambda, \Lambda)$.

3.4.2. Definition. Irreducible representation $\pi: \mathbb{C}[K]_{q} \rightarrow$ End $H$ is said to correspond to Schubert cell $X_{w}$ (see 1.3.5) provided for each $\Lambda \in P_{+}$the following conditions hold: a) $\pi\left(\mathscr{J}_{0}(w, \Lambda)\right)=0$; b) $\pi\left(C_{-w \Lambda, \Lambda}^{\Lambda}\right) \neq 0$.

3.4.3. Theorem. [29]. Each irreducible representation $\pi$ of algebra $\mathbb{C}[K]_{q}$ corresponds to unique Schubert cell $X_{w}$.

In the rest of Subsect. 3.4 we prove Theorem 3.4.3.

Proposition 3.2.1 implies the existence of $\Lambda \in P_{+}$such that $\pi\left(A_{\Lambda}\right) \neq 0$. Hence, there exists the matrix element $C_{-\mu, i, \Lambda}^{\Lambda}$ such that $\pi\left(C_{-\mu, i, \Lambda}^{\Lambda}\right) \neq 0$. Consider the set $\mathscr{D}_{0}(\Lambda)$ consisting of $\mu \in P(\Lambda)$ such that there exists the matrix element $C_{-\mu, j, \Lambda}^{\Lambda}$ with the property $\pi\left(C_{-\mu, j, \Lambda}^{\Lambda}\right) \neq 0$. Our considerations imply $\mathscr{D}_{0}(\Lambda) \neq \emptyset$. Let $\mathscr{D}_{1}(\Lambda)$ be the set of minimal elements of $\mathscr{D}_{0}(\Lambda)$ with respect to the usual partial order on weights (see $3.2 \mathrm{~b})$ ). We have $\mathscr{D}_{1}(\Lambda) \neq \emptyset$ and if $\lambda \in \mathscr{D}_{1}(\Lambda)$ then $\pi\left(\mathscr{J}_{2}(\lambda, \Lambda)\right)=0$.

From Propositions 2.8 and 3.4.1 it follows that

$$
\pi\left(C_{-\lambda, i, \Lambda}^{\Lambda}\right)^{*} \pi\left(C_{-\lambda, i, \Lambda}^{\Lambda}\right)=q^{-(\Lambda, \Lambda)+(\lambda, \lambda)} \pi\left(C_{-\lambda, i, \Lambda}^{\Lambda}\right) \pi\left(C_{-\lambda, i, \Lambda}^{\Lambda}\right)^{*} .
$$

Since $q>1$, this and the boundedness of $\pi\left(C_{-\lambda, i, \Lambda}^{\Lambda}\right)$ yield $-(\Lambda, \Lambda)+(\lambda, \lambda)=0$. Therefore, $\lambda=w \Lambda$ for some $w \in W$ and $C_{-\lambda, i, \Lambda}^{\Lambda}=C_{-w \Lambda, \Lambda}^{\Lambda}$. Moreover, $\pi\left(C_{-w \Lambda, \Lambda}^{\Lambda}\right)$ is a normal operator. 
3.4.4. Proposition. $\operatorname{Ker} \pi\left(C_{-w \Lambda, \Lambda}^{\Lambda}\right)=0$.

Proof. This follows from 3.4.1 and irreducibility of $\pi$.

3.4.5. Proposition. The spectrum of the operator $\pi\left(C_{-w \Lambda, A}^{\Lambda}\right)$ is of the form $\sigma\left(\pi\left(C_{-w \Lambda}^{\Lambda}, A\right)\right)=E \cup\{0\}$, where $E$ is a bounded set having no limit points in $\mathbb{C} \backslash 0$.

Proof. Let $Z_{0} \in \sigma\left(\pi\left(C_{-w \Lambda, \Lambda}^{\Lambda}\right)\right) \backslash 0$. From 3.4.1 we can easily derive that there exists an open proper subset $\Omega \subset \mathbb{C}$ such that

a) $Z_{0} \in \Omega$.

b) $\Omega$ is invariant under the group generated by homotheties with coefficients $q^{\left(\Lambda, \omega_{i}\right)-(w \Lambda, \mu)}$, where $\left\{\omega_{i}\right\}_{i=1}^{m}$ is the set of fundamental weights of Lie algebra $g$ and $\mu \in P\left(\omega_{i}\right)$.

Let $E_{\Omega}$ be the spectral projection of operator $\pi\left(C_{-w \Lambda, \Lambda}^{\Lambda}\right)$. From 3.2.1 and 3.4.1 it follows that $E_{\Omega} \pi(f)=\pi(f) E_{\Omega}$ for all $f \in \mathbb{C}[K]_{q}$. We conclude that $E_{\Omega}=C \cdot$ Id, where $C \in \mathbb{C}$. Now it is easy to understand that $Z_{0}$ is a point of the discrete spectrum and that $0 \in \sigma\left(\pi\left(C_{-w \Lambda, \Lambda}^{\Lambda}\right)\right)$. From this and properties a), b) the description of the spectrum follows.

Since $\pi\left(C_{-w \Lambda, \Lambda}^{\Lambda}\right)$ is a normal operator, from 3.4 .5 it follows that $H$ can be decomposed as follows:

$$
H=\bigoplus_{\gamma \in E} H_{\gamma}(w, \Lambda), \quad \text { where } \quad H_{\gamma}(w, \Lambda)=\left\{x \in H \mid \pi\left(C_{-w \Lambda, \Lambda}^{\Lambda}\right) x=\gamma x\right\} .
$$

In $E$, we introduce the following partial order: $\gamma_{1} \geqq \gamma_{2}$ if and only if $\left|\gamma_{1}\right| \geqq\left|\gamma_{2}\right|$. From 3.4.5 it follows that there exists the maximal element $\gamma_{0}=\gamma_{0}(w, \Lambda)$ with respect to this order.

3.4.6. Proposition. Let $v \in H_{\gamma_{0}}(w, \Lambda)$. Then for every matrix element $C_{-\mu, i, \Lambda}^{\Lambda}$ with $\mu \neq w \Lambda$ we have $\pi\left(C_{-\mu, i, \Lambda}^{\Lambda}\right) v=0$.

Proof. From 3.4.1 it follows that if $\pi\left(C_{-\mu, i, \Lambda}^{\Lambda}\right) v \neq 0$ the it is an eigenvector of $\pi\left(C_{-w \Lambda, \Lambda}^{\Lambda}\right)$ with the eigenvalue $\gamma_{0} q^{(\Lambda, \Lambda)-(w \Lambda, \mu)}>\gamma_{0}$. But this contradicts the maximality of $\gamma_{0}$.

So far the weight $\Lambda \in P_{+}$was assumed fixed. Hence $w \in W$ depended on $\Lambda$. Now we omit this assumption.

3.4.7. Proposition . Let $\Lambda_{1}, \Lambda_{2} \in P_{++}$satisfy the condition $\pi\left(A_{\Lambda_{\imath}}\right) \neq 0, i=1,2$, and let $w_{i} \in W$ be determined as in 3.4.3. Then $w_{1}=w_{2}$.

Proof. $\left(\Lambda_{1}, \Lambda_{2}\right)=\left(w_{1} \Lambda_{1}, w_{2} \Lambda_{2}\right)$ implies $w_{1}=w_{2}$.

From 3.2.1b) it follows that there exists a weight satisfying the conditions of Proposition 3.4.7. Let $w \in W$ be the corresponding element in the Weyl group. It is easy to see that the following result holds.

3.4.8. Proposition. $\pi\left(C_{-w \omega_{i}, \omega_{i}}^{\omega_{i}}\right) \neq 0$ for all fundamental weights $\omega_{i}, 1 \leqq i \leqq m$.

By Proposition 3.4.8, $\pi\left(C_{-w \lambda, \lambda}^{\lambda}\right) \neq 0 \forall \lambda \in P_{+}$.

3.4.9. Proposition. For every $\Lambda \in P_{+}$the set $\mathscr{D}_{1}(\Lambda)$ (see 3.4.3) consists of one element.

Proof. Let $w_{1} \Lambda \in \mathscr{D}_{1}(\Lambda), w_{1} \Lambda \neq w \Lambda$. From our considerations it follows that by 3.4.4 $\operatorname{Ker} \pi\left(C_{-w_{1} \Lambda, \Lambda}^{\Lambda}\right)=0$ and by 3.4.6 $\operatorname{Ker} \pi\left(C_{-w_{1} \Lambda, \Lambda}^{\Lambda}\right) \neq 0$. Hence, $w_{1} \Lambda=w \Lambda$. 
3.4.10. In this subsection, we conclude the proof of Theorem 3.4.3. It remains to show that $\pi\left(\mathscr{J}_{0}(w, \Lambda)\right)=0$. Set $E_{w}(\Lambda)=U_{q}\left(b_{+}\right) v_{w \Lambda}$ and consider a matrix element $C_{-\mu, i, \Lambda}^{\Lambda}$. If $\mu<w \Lambda$, then $C_{-\mu, i, \Lambda}^{\Lambda} \in \mathscr{J}_{2}(w, \Lambda)$ and $\pi\left(C_{-\mu, i, \Lambda}^{\Lambda}\right)=0$ (see 3.4.3). Further, if $\mu \nsupseteq w \Lambda$ and $\mu \nless w \Lambda$, then $\pi\left(C_{-\mu, i, \Lambda}^{\Lambda}\right)=0$ as well. For proof, we can choose a linear ordered chain of weights of $\mathscr{D}_{0}(\Lambda)$ which contains $\mu$. The minimal element of this chain belongs to $\mathscr{D}_{1}(\Lambda)$ and differs from $w \Lambda$, but this contradicts 3.4.9. The last case, when $\mu>w \Lambda$ but $v_{\mu}^{(i)} \notin E_{w}(\Lambda)$, can be considered in complete analogy with the second one [one should use (3.1) and Proposition 3.4.6]. We obtain $\pi\left(C_{-\mu, i, \Lambda}^{\Lambda}\right)=0$ once more and the proof of Theorem 3.4.3 is completed.

3.5. Let $\gamma_{0}(w, \Lambda)$ be the eigenvalue determined in 3.4 .5 .

3.5.1. Proposition. $\left|\gamma_{0}(w, \Lambda)\right|=1$.

Proof. Let $T_{A}$ be a matrix consisting of the matrix elements of representation $\varrho_{A}: U_{q}(g) \rightarrow$ End $L(\Lambda)$ in orthonormal weight basis. It is easy to show that $\left(T_{\Lambda}^{t}\right)^{*} T_{A}$ $=T_{\Lambda}\left(T_{A}^{t}\right)^{*}=1$, where $\left(T_{A}^{t}\right)^{*}$ is obtained by action of involution on each element of the transposed matrix $T_{\Lambda}^{t}$. It follows that

$$
\sum_{\mu, i}\left(C_{-\mu, i, \Lambda}^{\Lambda}\right)^{*} C_{-\mu, i, \Lambda}^{\Lambda}=1,
$$

and it remains to use Proposition 3.4.6.

3.5.2. Remark. From Proposition 3.5.1, it follows that if $\pi: \mathbb{C}[K]_{q} \rightarrow$ End $V$ is a representation in a preHilbert space $V$ it extends to a representation in $H=\bar{V}$, where $\bar{V}$ is the completion of $V$.

3.5.3. Proposition. $\operatorname{dim} H_{\gamma_{0}(w, \Lambda)}(w, \Lambda)=1$.

Proof. Let $\mathscr{U}$ be a commutative $*$-subalgebra in End $H$ generated by normal operators $\pi\left(C_{-w \lambda, \lambda}^{\lambda}\right), \lambda \in P_{+}$. For fixed $\Lambda \in P_{+}$we have $\mathscr{U}\left(H_{\gamma_{0}(w, \Lambda)}(w, \Lambda)\right)$ $C H_{\gamma_{0}(w, \Lambda)}(w, \Lambda)$ and to complete the proof we use 3.4.1, 3.5.1 and the irreducibility of $\pi$.

3.5.4. Corollary. $H_{\gamma_{0}(w, \Lambda)}(w, \Lambda)$ is independent of $\Lambda$.

Proof. This follows from 3.5.1, 3.5.3 and commutativity of $\mathscr{U}$.

3.5.5. Let $H_{\gamma_{0}}(w)$ denote $H_{\gamma_{0}(w, \Lambda)}(w, \Lambda)$. Since $H_{\gamma_{0}}(w)=\mathbb{C} v^{+}$, where $v^{+} \in H$, we have $\pi(f) v^{+}=\chi(f) v^{+}$for all $f \in A_{\Lambda}$, where $\chi(f) \in \mathbb{C}$. Clearly, $\chi$ determines the homomorphism of $A_{+}$into $\mathbb{C}$. From Propositions 3.4.6 and 3.5.3, we complete the proof of Theorem 3.3.

3.5.6. Definition. The homomorphism $\chi: A_{+} \rightarrow \mathbb{C}$ is called the highest weight of irreducible representation $\pi$.

3.5.7. Remark. One can show that a space $H$ of irreducible representation of $\mathbb{C}[K]_{q}$ is a completion of a unitarizable simple $\mathbb{C}[G]_{q}$-module $V$. It is easy to show that this gives the bijection "space $H \leftrightarrow$ module $V$ ".

3.5.8. Proposition. The irreducible representations of $\mathbb{C}[K]_{q}$ are equivalent iff their highest weights are equal.

Proof is analogous to that of Theorem 1b), in Part III, Chap. VII, Sect. 3 of [25]. In the course of the proof Remark 3.5.7 is used. 
3.5.9. Remark. a) Considerations from previous subsections show that in the representation theory of the algebra $\mathbb{C}[K]_{q}$ there is an analog of Borel subalgebra (i.e. $A_{+}$) but no analog of Cartan subalgebra. Nevertheless, for every $w \in W$ there exists an analog of Cartan subalgebra, i.e. *-subalgebra generated by $C_{-}^{\omega_{i}}{ }_{w \omega_{i}, \omega_{i}}$, $1 \leqq i \leqq m$ (see 3.5.3).

b) There is further analogy between representation theory of $\mathbb{C}[K]_{q}$ and that of $U(g)$ : one can define "Verma modules over $\mathbb{C}[G]_{q}$ " and develop the theory of the highest weight modules over $\mathbb{C}[G]_{q}$. In particular, this allows to construct the irreducible representations of $\mathbb{C}[K]_{q}$ with given highest weights [28]. In the next subsections, we'll develop another method of construction of representations.

3.6. Let $i$ be a fixed vertex of Dynkin diagram of the Lie algebra $g$. $U_{q}\left(\operatorname{sl}(2)_{i}\right)$ denotes the Hopf $*$-subalgebra in $U_{q}(g)$ generated by $X_{i}^{+}, X_{i}^{-}, k_{i}^{+}, k_{i}^{-}$. Let $\varphi_{i}: U_{q}\left(\operatorname{sl}(2)_{i}\right) \rightarrow U_{q}(g)$ be embedding and $\varphi_{i}^{*}: \mathbb{C}[K]_{q} \rightarrow \mathbb{C}\left[S U(2)_{i}\right]_{q}$ be the corresponding epimorphism of the algebra of functions. Recall that irreducible representations of $\mathbb{C}\left[S U(2)_{i}\right]_{q}$ were described in $[31]$ where $\ell_{2}\left(\mathbb{Z}_{+}\right)$-realizations of the former were obtained. In [31] it was proved that irreducible representations of $\mathbb{C}\left[S U(2)_{i}\right]_{q}$ are parameterized by simplex leaves of Poisson group $S U(2)$. Let

$$
\mathscr{D}=\left\{\left(\begin{array}{cc}
a & -b \\
b & \bar{a}
\end{array}\right) \mid b<0\right\}
$$

be a symplectic leaf in $S U(2)$ through the point $\left(\begin{array}{cc}0 & 1 \\ -1 & 0\end{array}\right)$ and let $\pi_{\mathscr{D}}: \mathbb{C}\left[S U(2)_{i}\right]_{q}$ $\rightarrow$ End $\ell_{2}\left(\mathbb{Z}_{+}\right)$be the corresponding representation (in formula (3.2) of [31], this representation was denoted by $\varrho_{\pi}$ ). Set $\pi_{s_{i}}=\pi_{\mathscr{D}} \circ \varphi_{i}^{*}$, where $s_{i} \in W$ is a simple reflection. It is easy to see that $\pi_{s_{i}}$ is an irreducible representation of algebra $\mathbb{C}[K]_{q}$, corresponding to Schubert cell $X_{s_{i}}$.

3.6.1. Proposition [29]. Let $w \in W$ satisfy $s_{i} w>w$ and let $\pi$ be irreducible and corresponds to the Schubert cell $X_{w}$. Then $\pi_{s_{i}} \otimes \pi$ is irreducible and corresponds to the Schubert cell $X_{s_{2} w}$.

We'll give the scheme of the proof.

Step 1. Let $\Lambda \in P_{++}$and $C_{-\mu, \kappa, \Lambda}^{\Lambda} \in \mathscr{J}_{0}\left(S_{i} w \Lambda, \Lambda\right)$. Consider the following equality

$$
\left(\pi_{s_{i}} \otimes \pi\right)\left(C_{-\mu, K, \Lambda}^{\Lambda}\right)=\sum_{\lambda, j} \pi_{s_{i}}\left(C_{-\mu, K, \lambda, j}^{\Lambda}\right) \otimes \pi\left(C_{-\lambda, j, \Lambda}^{\Lambda}\right) .
$$

Since $U_{q}\left(b_{+}\right)$-module $E_{s_{i} w}(\Lambda)=U_{q}\left(b_{+}\right) V_{s_{i} w \Lambda}$ is invariant under $X_{i}^{ \pm}$, it follows that the right side in (3.2) is zero. Hence, $\left(\pi_{s_{i}} \otimes \pi\right)\left(\mathscr{J}_{0}\left(s_{i} w \Lambda, \Lambda\right)=0\right.$ for all $\Lambda \in P_{++}$.

Step 2. In (3.2), set $\mu=s_{i} w \Lambda$. Then the arguments of Step 1 give

$$
\left(\pi_{s_{i}} \otimes \pi\right)\left(C_{-s_{i} w \Lambda, \Lambda}^{\Lambda}\right)=\pi_{s_{i}}\left(C_{-s_{i} w \Lambda, w \Lambda}^{\Lambda}\right) \otimes \pi\left(C_{-w \Lambda, \Lambda}^{\Lambda}\right) .
$$

From Sect. 6 of [31], we can compute explicitly $\varphi_{i}^{*}\left(C_{-s_{i} w \Lambda, A}^{\Lambda}\right)$ and show that $\pi_{s_{i}}\left(C_{-s_{i} w A, w A}^{\Lambda}\right) \neq 0$.

Step 3. Now we can prove the irreducibility of $\pi_{S_{i}} \otimes \pi$. Let $H_{w_{1}} C H_{S_{i}} \otimes H$ be a subspace of irreducible representation corresponding to Schubert cell $X_{w_{1}}, w_{1} \in W$. Clearly, the only case $w_{1} \leqq s_{i} w$ is possible. Let $\left\{e_{K}\right\}_{K \geqq 0}$ be the standard orthonormal basis in $H_{s_{i}}=\ell_{2}\left(\mathbb{Z}_{+}\right)$and $\left\{f_{m}\right\}_{m \geqq 0}$ be an orthonormal basis in $H$. From (3.2) and formulae (3.2) of [7], it follows that $\left\{e_{k} \otimes f_{m}\right\}_{k, m \geqq 0}$ is a complete 
system of eigenvectors of the operator $\left(\pi_{s_{i}} \otimes \pi\right)\left(C_{-s_{i} w \Lambda, \Lambda}^{\Lambda}\right)$. From (3.3), it is now easy to derive that the condition $w_{1}<s_{i} w$ doesn't hold. Comparing the modules of eigenvalues of the operator $\left(\pi_{s_{i}} \otimes \pi\right)\left(C_{-s_{i} w \Lambda, \Lambda}^{\Lambda}\right)$ we see that in the case $w_{1}=s_{i} w$ there exists unique subrepresentation $H_{s_{i} w} \subset H_{s_{l}} \otimes H_{w}$, corresponding to Schubert cell $X_{s_{i} w}$. Hence, $H_{s_{i} w}=H_{s_{i}} \otimes H_{w}$.

3.6.2. Corollary. If $w=s_{i_{1}} \ldots s_{i_{K}}$ is a reduced expression in Weyl group $W$ then the representation $\pi_{s_{i_{1}}} \otimes \ldots \otimes \pi_{s_{i_{K}}}$ is irreducible and corresponds to Schubert cell $X_{w_{w}}$.

It is also easy to prove two following propositions.

3.6.3. Proposition. Let $\tau: \mathbb{C}[K]_{q} \rightarrow \mathbb{C}$ be one-dimensional representation. Then $\tau$ corresponds to Schubert cell $X_{e}$, where $e$ is the unit in $W$. All one-dimensional representations are parameterized by points of maximal torus $T \subset K$.

3.6.4. Proposition. If irreducible representation $\pi$ corresponds to Schubert cell $X_{w}$ and $\tau$ is one-dimensional representation, then the representations $\pi \otimes \tau$ and $\tau \otimes \pi$ are irreducible and correspond to Schubert cell $X_{w}$.

Let $i, j$ be two vertices of Dynkin diagram and let $m_{i j}$ be the order of the element $s_{i} s_{i}$ in Weyl group $W$.

3.6.5. Proposition [29]. Representations $\pi_{s_{i}} \otimes \pi_{s_{j}} \otimes \pi_{s_{i}} \otimes \ldots$ and $\pi_{s_{j}} \otimes \pi_{s_{i}} \otimes \pi_{s_{j}} \otimes \ldots$ ( $m_{i j}$ factors in each expression) are isomorphic.

For proof compare the highest weights of these representations.

3.6.6. Corollary. For each reduced expression $w=s_{i_{1}} \ldots s_{i_{K}}$ the representation $\pi_{w}$ $=\pi_{s_{i_{1}}} \otimes \ldots \otimes \pi_{s_{i_{K}}}$ is irreducible and independent of (up to isomorphism) the choice of reduced expression.

Proof. This is a corollary of 3.6.2, 3.6.5.

We can unite the results of previous sections and obtain all irreducible representations of $\mathbb{C}[K]_{q}$.

3.6.7. Theorem. a) For every irreducible representation $\pi$ of $\mathbb{C}[K]_{q}$ there exist unique $w \in W$ and unique $t \in T$ such that $\pi$ is isomorphic to a representation of the form $\pi_{s_{i_{1}}} \otimes \ldots \otimes \pi_{s_{i_{K}}} \otimes \tau_{t}$, where $w=s_{i_{1}} \ldots s_{i_{K}}$ is a reduced expression and $\tau_{t}$ is onedimensional representation, corresponding to a point $t$.

b) The set of irreducible representations corresponding to Schubert cell $X_{w}$ is of the form $\left\{\pi_{w} \otimes \tau_{t}\right\}_{t \in T}$.

3.6.8. Remark. Theorem 3.6.7 is the quantum analog of Propositions 1.3.1 and 1.3.3.

3.7. Consider Gelfand-Naimark-Segal state corresponding to representation $\pi_{S_{i}}$ and vector $e_{0}$ :

$$
\bar{s}_{i}(f)=\left(\pi_{S_{i}}(f) e_{0}, e_{0}\right) .
$$

Clearly, $\bar{s}_{i} \in \mathbb{C}[K]_{q}^{V}$, where $\mathbb{C}[K]_{q}^{V}$ is the Hopf algebra dual to $\mathbb{C}[K]_{q}$.

3.7.1. Proposition. For $i \neq j$ the following "quantum Coxeter relation" hold

$$
\bar{s}_{i} \bar{s}_{j} \bar{s}_{i} \ldots=\bar{s}_{j} \bar{s}_{i} \bar{s}_{j} \ldots
$$


( $m_{i j}$ factors in each product), where $m_{i j}$ is the order of the element $s_{i} s_{j}$ in (usual) Weyl group.

3.7.2. Remark. In Sect. 5, we'll use Proposition 3.7.1 in construction of the quantum analog of the Weyl group. For the case $K=S U(2)$, the quantum Weyl group was constructed in [26] (see also [31]).

\section{Representations of Twisted Algebras of Functions and Quantum Tori}

4.1. In this section we investigate irreducible representations of algebras $\mathbb{C}\left[K^{u}\right]_{q}$ $=\mathbb{C}[K(1, u)]_{q}$ and $\mathbb{C}[K(0, u)]_{q}$ in Hilbert space. The corresponding theorems are the quantum analogs of 1.4.3 and 1.5.2. Most considerations in the case of $\mathbb{C}\left[K^{u}\right]_{q}$ are in analogy with Sect. 3, the appearance of the quantum tori being due to the non-commutativity of the subalgebra generated by $\pi\left(C_{-{ }_{-w} \omega_{i}, \omega_{l}}^{\omega_{2}}\right), 1 \leqq i \leqq m$ [see (3.5.9)]. This subalgebra was commutative in Sect. 3 and now it is a quantum torus.

In the case of $\mathbb{C}[K(0, u)]_{q}$ quantum tori appear in a similar way.

We do not know whether there exist analogs of theorems of Subsect. 3.6 and the "twisted" quantum Weyl group.

First we consider representations of $\mathbb{C}\left[K^{u}\right]_{q}$ and then those of $\mathbb{C}[K(0, u)]_{q}$.

We adopt notations from Sect. 3 .

4.2. Note that in $\mathbb{C}\left[K^{u}\right]_{q}$ all the ideals from 3.4 are well-defined. Definition 3.4 .2 also makes sense in $\mathbb{C}\left[K^{u}\right]_{q}$. In complete analogy with 3.4 , the following theorem can be proved.

4.2.1 Theorem. Every irreducible representation $\pi$ of $\mathbb{C}\left[K^{u}\right]_{q}$ corresponds to unique Schubert cell $X_{w}$.

In the proof, we use the following proposition instead of 3.4.1.

4.2.2. Proposition. In $\mathbb{C}\left[K^{u}\right]_{q} / \mathscr{J}_{2}(\lambda, \Lambda)$, the following relation holds:

$$
C_{-\mu, \gamma, \Omega}^{v} \circ C_{-\lambda, j, \Lambda}^{\Lambda}=q^{-(\Lambda, \gamma)+(\lambda, \mu)+i(\Lambda, \gamma)_{u}-i(\lambda, \mu)_{u}} C_{-\lambda, j, \Lambda}^{\Lambda} \circ C_{-\mu, \gamma, \Omega}^{v},
$$

where $(x, y)_{u}$ was defined in 2.7 .

4.2.3. Corollary. Let a representation $\pi$ of $\mathbb{C}\left[K^{u}\right]_{q}$ correspond to Schubert cell $X_{w}$. Then for every $\lambda, \mu \in P_{+}$the following relation holds:

$$
\pi\left(C_{-w \lambda, \lambda}^{\lambda}\right) \pi\left(C_{-w \mu, \mu}^{\mu}\right)=q^{i(\lambda, \mu)_{u}-i(w \lambda, w \mu)_{u}} \pi\left(C_{-w(\lambda+\mu), \lambda+\mu}^{\lambda+\mu}\right) .
$$

4.3. Let $\mathscr{J}_{q, u, w}$ be minimal two-sided *-ideal in $\mathbb{C}\left[K^{u}\right]_{q}$, containing all ideals $\mathscr{J}_{o}(w \Lambda, \Lambda), \Lambda \in P_{+}$. Set $\mathbb{C}\left[K^{u}\right]_{q, w}=\mathbb{C}\left[K^{u}\right]_{q} / \mathscr{J}_{q, u, w}$. Clearly, there is a bijection between the set of irreducible representations $\pi$ of $\mathbb{C}\left[K^{u}\right]_{q, w}$, corresponding to $X_{w}$ and that of irreducible representations $\pi$ of $\mathbb{C}\left[K^{u}\right]_{q, w}$ such that

$$
\operatorname{Ker} \pi\left(C_{-w \lambda, \lambda}^{\lambda}\right)=0 \quad \forall \lambda \in P_{+} .
$$

In the theory of irreducible representations of $\mathbb{C}\left[K^{u}\right]_{q}$, a unital *-subalgebra $P_{q, u, w}$ $\subset \mathbb{C}\left[K^{u}\right]_{q, w}$, generated by elements $C_{\lambda}=C_{-w \lambda, \lambda}^{\lambda}, \lambda \in P_{+}$, plays the part of Cartan subalgebra (we use the notation $C_{-w \lambda, \lambda}^{\lambda}$ for image of $C_{-w \lambda, \lambda}^{\lambda} \in \mathbb{C}\left[K^{u}\right]_{q}$ in the factoralgebra $\left.\mathbb{C}\left[K^{u}\right]_{q, w}\right)$. 
For $\lambda, \mu \in P_{+}$, set

$$
\begin{gathered}
\theta_{w, u}(\lambda, \mu)=(\lambda, \mu)_{u}-(w \lambda, w \mu)_{u}, \\
\sigma(\lambda, \mu)=\sigma_{q, u, w}(\lambda, \mu)=q^{i \theta_{w, u}(\lambda, \mu)} .
\end{gathered}
$$

4.3.1. Proposition. a) In algebra $P_{q, u, w}$, the following relations hold:

$$
\begin{aligned}
& C_{\lambda} C_{\mu}=\sigma(\lambda, \mu) C_{\lambda+\mu}, \\
& C_{\lambda} C_{\mu}=\sigma(\lambda, \mu)^{2} C_{\mu} C_{\lambda}, \\
& C_{\lambda} C_{\mu}^{*}=\sigma(\mu, \lambda)^{2} C_{\mu}^{*} C_{\lambda} .
\end{aligned}
$$

b) In $P_{q, u, w}$, the elements $\mathscr{D}_{\lambda}=C_{\lambda}^{*} C_{\lambda}=C_{\lambda} C_{\lambda}^{*}$ are central.

Proof. See (2.13) and Subsects. 2.8.1, 4.2.2.

From 4.2 .2 , it follows that algebra $P_{q, u, w}$ is generated by $C_{j}=C_{\omega_{j}}, 1 \leqq j \leqq m$.

The following result is immediate.

4.3.2. Proposition. In $\mathbb{C}\left[K^{u}\right]_{q, w}$, the following commutation relations between $\mathscr{D}_{j}$ $=\mathscr{D}_{\omega_{j}}$ and matrix elements hold:

$$
\begin{gathered}
\mathscr{D}_{j} C_{-\mu, P, \lambda}^{\lambda}=q^{2 \kappa_{j}(\lambda, \mu)} C_{-\mu, P, \lambda}^{\lambda} \mathscr{D}_{j}, \\
\mathscr{D}_{j}\left(C_{-\mu, P, \lambda}^{\lambda}\right)^{*}=q^{-2 \kappa_{j}(\lambda, \mu)}\left(C_{-\mu, P, \lambda}^{\lambda}\right)^{*} \mathscr{D}_{j},
\end{gathered}
$$

where $\kappa_{j}(\lambda, \mu)=\left(\omega_{j}, \lambda\right)-\left(w \omega_{j}, \mu\right) \geqq 0$.

Note that for a given $\operatorname{par}(\lambda, \mu)$ with $\mu \neq w \lambda$ there exists $j$ such that $\kappa_{j}(\lambda, \mu)>0$.

4.4. Let $\pi: \mathbb{C}\left[K^{u}\right]_{q} \rightarrow$ End $H$ be irreducible representation corresponding to a Schubert cell $X_{w}$. With analogy to 3.4 .5 , one can show that the spectrum of the selfadjoint operator $\pi\left(\mathscr{D}_{j}\right)$ is $\{0\} \cup E_{j}$, where $E_{j}$ is a discrete set of eigenvalues with 0 as the unique limit point.

A tuple $\gamma=\left(\gamma_{1}, \ldots, \gamma_{m}\right) \in E \stackrel{\text { def }}{=} E_{1} \times \ldots \times E_{m}$ will be called a weight of representation $\pi$.

Note that $E$ is partially ordered: $\gamma \geqq \gamma^{\prime}$ iff $\gamma_{j} \geqq \gamma_{j}^{\prime}$ for all $j \in[1, m]$.

4.4.1. Definition. In $E$, a maximal element with respect to this ordering is called a maximal weight of representation $\pi$.

Let $H_{\gamma}=\left\{V \in H \mid \pi\left(\mathscr{D}_{j}\right) V=\gamma_{j} V\right\}$ be a weight space of the weight $\gamma$. We have $H=\bigoplus_{\gamma \in E} H_{\gamma}$. Let $\gamma^{0}$ be a maximal weight of representation $\pi$. Let $\gamma_{j}^{0}$ be the maximal eigenvalue of $\pi\left(\mathscr{D}_{j}\right)$ and let $H\left(\gamma_{j}^{0}, \mathscr{D}_{j}\right)$ be the corresponding eigenspace.

4.4.2. Proposition. For every $j \in[1, m]$,

a) algebra $P_{q, u, w}$ acts irreducibly in $H\left(\gamma_{j}^{0}, \mathscr{D}_{j}\right)$,

b) $H\left(\gamma^{0}\right)=H\left(\gamma_{j}^{0}, \mathscr{D}_{j}\right)$.

Proof. a) Let $\hat{H} \subset H\left(\gamma_{j}^{0}, \mathscr{D}_{j}\right)$ be invariant under $\pi\left(P_{q, u, w}\right)$. From 4.3 .2 , it is easy to derive that

$$
\pi\left(\mathbb{C}\left[K^{u}\right]_{q, w}\right)(\hat{H}) \subset \hat{H} \oplus \sum_{\gamma_{s} \leqq \gamma_{j}^{0}} H\left(\gamma_{s}, \mathscr{D}_{j}\right),
$$

where $H\left(\gamma_{s}, \mathscr{D}_{j}\right)$ is the eigenspace of $\pi\left(\mathscr{D}_{j}\right)$, corresponding to eigenvalue $\gamma_{s}$. Since $\pi$ is irreducible, $\hat{H}=H\left(\gamma_{j}^{0}, \mathscr{D}_{j}\right)$. 
b) Due to a),

$$
H\left(\gamma_{j}^{0}, \mathscr{D}_{j}\right)=\pi\left(P_{q, u, w}\right) v \quad \forall v \in H\left(\gamma_{j}^{0}, \mathscr{D}_{j}\right)
$$

and due to 4.3.1, $H=\pi\left(P_{q, u, w}\right) \oplus H_{1}$, where $H_{1}$ denotes a subspace spanned by vectors of weights $\gamma<\gamma_{j}^{0}$. Now, let $j_{i} \neq j . H\left(\gamma_{j}^{0}, \mathscr{D}_{j}\right)$ being invariant under $\pi\left(\mathscr{D}_{j_{1}}\right)$, we can assume $V \in H\left(\gamma_{j_{1}}^{0}, \mathscr{D}_{j_{1}}\right)$ (from 4.3.2 it follows that if $v \in H\left(\gamma_{j_{1}}^{\prime}, \mathscr{D}_{j_{1}}\right)$, then $\left.H \subset \underset{\gamma^{\prime} \leqq \gamma_{j_{1}}}{\bigoplus} H\left(\gamma^{\prime}, \mathscr{D}_{j_{1}}\right)\right)$. Hence,

$$
H\left(\gamma_{j_{1}}^{0}, \mathscr{D}_{j_{1}}\right)=\pi\left(P_{q, u, w}\right) v \subset H\left(\gamma_{j}^{0}, \mathscr{D}_{j}\right)
$$

By substitution $j_{1}$ for $j$ and vice versa we obtain

$$
H\left(\gamma_{j}^{0}, \mathscr{D}_{j}\right)=H\left(\gamma_{j_{1}}^{0}, \mathscr{D}_{j_{1}}\right)=H\left(\gamma^{0}\right) \text {. }
$$

4.4.3. Proposition. The maximal weight of the irreducible representation $\pi$ is equal to $\gamma^{0}=(1,1, \ldots, 1)$.

Proof is analogous to that of Proposition 3.5.1, use being made of Propositions 4.3.2, 4.4.2b) and the following formula:

$$
\pi\left(\mathscr{D}_{\lambda}\right)=\pi\left(C_{\lambda}^{*}\right) \pi\left(C_{\lambda}\right)=I \quad \text { on } \quad H\left(\gamma^{0}\right) .
$$

4.5. Recall the definition of quantum tori (see, for example, the review [23]). Let $P$ be an abelian group and $\kappa: P \times P \rightarrow \mathbb{R}$ be antisymmetric bilinear form. Set $\sigma(\lambda, \mu)$ $=\exp (i \kappa(\lambda, \mu)) . A(P, \sigma)$ denotes the unital *-algebra with generators $U_{\lambda}, \lambda \in P$, and defining relations

$$
\begin{gathered}
U_{\lambda}^{*} U_{\lambda}=U_{\lambda} U_{\lambda}^{*}=I, \\
U_{\lambda} U_{\mu}=\sigma(\lambda, \mu) U_{\lambda+\mu} .
\end{gathered}
$$

4.5.1. Let $\mathscr{J}$ denote two-sided $*$-ideal in $P_{q, u, w}$ generated by $\mathscr{D}_{j}-1, j=1, \ldots, m$. From 4.3.1 and (4.4) it follows that irreducible representation $\pi: \mathbb{C}\left[K^{u}\right]_{q, w} \rightarrow$ End $H$ satisfying the condition (4.1), determines unique irreducible representation $\chi$ of factor-algebra $P_{q, u, w} / \mathscr{J}$ in $H\left(\gamma^{0}\right)$. Also, $P_{q, u, w} / \mathscr{J} \cong A(P, \sigma)$, where $P$ is the integer root lattice and $\sigma=\sigma_{q, u, w}$ is defined by (4.2), (4.3).

4.5.2. Definition. The $*$-representation $\chi: A(P, \sigma) \rightarrow$ End $H\left(\gamma^{0}\right)$ is called the highest weight of irreducible representation $\pi$.

4.5.3. Proposition [13]. Irreducible representations corresponding to the Schubert cell $X_{w}$ are equivalent iff their highest weights are equivalent.

4.5.4. Theorem [13]. For each $w \in W$ and for each irreducible representation $\chi$ of $A\left(P, \sigma_{q, u, w}\right)$ in a Hilbert space there exists the irreducible representation $\pi$, which corresponds to Schubert cell $X_{w}$ and has the highest weight $\chi$.

Proof. Set $\quad \tilde{P}=P \oplus P, \quad \tilde{\sigma}\left((\lambda, \mu),\left(\lambda^{\prime}, \mu^{\prime}\right)\right)=q^{i\left[\left(\mu, \mu^{\prime}\right)_{u}-\left(\lambda, \lambda^{\prime}\right)_{u}\right]}, \quad \mathbb{C}\left[K^{u}\right]_{q, w}^{\sim}$ $=\mathbb{C}\left[K^{0}\right]_{q, w} \otimes A(\tilde{P}, \tilde{\sigma})$ and introduce an involution in $\mathbb{C}\left[K^{u}\right]_{q, w}^{\tilde{y}}$ by $(a \otimes b)^{*}$ $=a^{*} \otimes b^{*}$. Further, define $*$-algebra embedding $j: \mathbb{C}\left[K^{u}\right]_{q, w} \rightarrow \mathbb{C}\left[K^{u}\right]_{q, w}^{\sim}$ by $j\left(C_{-\mu, i, \lambda, K}^{\omega}\right)=C_{-\mu, i, \lambda, K}^{\omega} \otimes U_{\lambda, \mu}$ and identify $A(P, \sigma)$ with subalgebra $A\left(P^{\prime}, \sigma^{\prime}\right)$ $C A(\widetilde{P}, \sigma)$, where $P^{\prime}=\{(-w \lambda, \lambda) \mid \lambda \in P\}, \sigma^{\prime}=\left.\tilde{\sigma}\right|_{P^{\prime} \times P^{\prime}}$. In the next subsection, we'll construct $*$-representation $\chi: A(\tilde{P}, \tilde{\sigma}) \rightarrow$ End $H$ such that $H_{\chi} \subset H_{\tilde{\chi}}$ and $\tilde{\chi}(f)=\chi(f)$ for all $f \in A(P, \sigma)$. Here $H_{\chi}\left(H_{\tilde{\chi}}\right)$ is the representation space of $\chi$ (of $\left.\tilde{\chi}\right)$. 
Let $\pi_{w}: \mathbb{C}\left[K^{0}\right]_{q, w} \rightarrow$ End $H$ be irreducible representation defined in 3.6.2. Then $\tilde{\pi}=\pi \otimes \chi$ is a representation of $\mathbb{C}\left[K^{u}\right]_{q, w}^{\sim}$ in $H \otimes H_{\tilde{\chi}}$. Denote by $\bar{H}$ the completion of the space $H \otimes H_{\tilde{\chi}}$. Let $\ell \in H$ be the unique $\pi\left(A_{+}\right)$-invariant straight line. Set $H^{\prime}$ $=\pi\left(\mathbb{C}\left[K^{u}\right]_{q, w}\right)\left(\ell \otimes H_{\tilde{\chi}}\right)$. This is a subspace in $\bar{H}$. From weight considerations, it is easy to show that in $H^{\prime}$ there exists unique $\pi\left(\mathbb{C}\left[K^{u}\right]_{q, w}\right.$-invariant proper subspace $H^{\prime \prime}$. Hence, $H^{\prime} \ominus H^{\prime \prime}$ is a space of irreducible representation we need.

The existence of $\tilde{\chi}$ follows from the next lemma.

4.5.5. Lemma. Let $P_{1}, P_{2} \subset \widetilde{P}$ be subgroups of abelian group $\widetilde{P}$ such that $\widetilde{P}=P_{1} \oplus P_{2}$, and let $A(\tilde{P}, \tilde{\sigma})$ and $A\left(P_{1}, \sigma_{1}\right)$ be quantum tori such that $\sigma_{1}=\left.\tilde{\sigma}\right|_{P_{1} \times P_{1}}$.

Then for every representation $\chi$ of $A\left(P_{1}, \sigma_{1}\right)$ in a Hilbert space $H_{\chi}$ there exist Hilbert space $H_{\tilde{\chi}} \supset H_{\chi}$ and representation $\tilde{\chi}: A(\widetilde{P}, \tilde{\sigma}) \rightarrow$ End $H_{\tilde{\chi}}$ such that $\chi(f)=\tilde{\chi}(f)$ for all $f \in A\left(P_{1}, \sigma_{1}\right)$.

Proof. Set $H=A(\tilde{P}, \tilde{\sigma}) \underset{A\left(P_{1}, \sigma_{1}\right)}{\bigotimes} H_{\chi}$, note that $H=\bigoplus_{\mu \in P_{2}} H_{\mu}$, where $H_{\mu}$ $=\left\{U_{\mu} \otimes x \mid x \in H_{\chi}\right\}$ and introduce inner product in $H$ as follows:

$$
\left\langle u_{\lambda} \otimes x, u_{\mu} \otimes y\right\rangle=\delta_{\lambda \mu}\langle x, y\rangle \quad \forall \lambda, \mu \in P_{2} .
$$

The completion $\bar{H}$ is the representation space we're looking for.

4.6. In this subsection, we discuss the relation between irreducible representations of $\mathbb{C}\left[K^{u}\right]_{q}$ and symplectic leaves of a Poisson Lie group $K^{u}=K(1, u)$ (see 1.4.3). First we obtain several results relevant to quantum tori.

Set $\sigma=\sigma_{q, u, w}, P_{Z}=\{\lambda \mid \sigma(\lambda, \mu)=1, \forall \mu \in P\}$ and denote by $Z(P, \sigma)$ the center of algebra $A(P, \sigma)$. An irreducible representation $\chi: A(P, \sigma) \rightarrow$ End $H$ determines the character $\chi_{Z}: Z(P, \sigma) \rightarrow \mathbb{C}$ but a character may correspond to several representations of $A(P, \sigma)$. Nevertheless, the following result holds.

4.6.1. Proposition. $\operatorname{Ker} \chi=A(P, \sigma)\left(\operatorname{Ker} \chi_{z}\right)$.

Proof. Let $u \in \operatorname{Ker} \chi \backslash A(P, \sigma)\left(\operatorname{Ker} \chi_{z}\right)$ be of the form $u=\sum_{\lambda \in M} d_{\chi} u_{\lambda}$ with the minimal number of non-zero summands. It is easy to show that there exist $\mu \in P, \lambda_{1}, \lambda_{2} \in M$ such that $\sigma\left(\lambda_{1}, \mu\right) \neq \sigma\left(\lambda_{2}, \mu\right)$ and the element

$$
u^{1}=u-\sigma\left(\lambda_{1}, \mu\right)^{-1} u_{-\mu} U u_{\mu} \in \operatorname{Ker} \chi \backslash A(P, \sigma)\left(\operatorname{Ker} \chi_{z}\right)
$$

is of the form

$$
u^{1}=\sum_{\lambda \in M \backslash \lambda_{1}} d_{\lambda}^{\prime} u_{\lambda} \quad \text { with } \quad d_{\lambda}^{\prime} \neq 0 .
$$

This contradicts the choice of $u$ and the proposition is proved.

Set $\hat{P}=P / P_{Z}$ and note that $\sigma$ determines $\hat{\sigma}: \hat{P} \times \hat{P} \rightarrow \mathbb{R}$. The following result is straightforward.

4.6.2. Proposition. $A$ character $\chi_{z}$ determines a $*$-isomorphism $\gamma: A(P, \sigma) / \operatorname{Ker} \chi_{Z}$ $\rightarrow A(\hat{P}, \hat{\sigma})$.

4.6.3. Proposition. A class of irreducible representations of algebra $A(\hat{P}, \hat{\sigma})$ is unique iff $\hat{P}$ is finite.

Proof. The center of $A(\hat{P}, \hat{\sigma})$ being trivial, the kernel of each irreducible representation is also trivial. If $\hat{P}$ is finite then $A(\hat{P}, \hat{\sigma})$ is a finite-dimensional $C^{*}$ algebra and $A(\hat{P}, \hat{\sigma})$ is of the type $\mathrm{I}[2]$, i.e. there is a natural bijection between 
classes of irreducible representations and primary ideals. If card $P=\infty$ then it easy to construct an example of non-equivalent irreducible representations of $A(\hat{P}, \hat{\sigma})$ (see e.g. [13]).

Thus, one can hope to find one-to-one correspondence between symplectic leaves and irreducible representations iff $\hat{P}$ is finite. Below the necessary and sufficient condition is given.

4.6.4. Proposition. Card $\hat{P}<\infty$ iff

$$
\ln q \theta_{u, w}(\lambda, \mu) \in \pi \mathbb{Q} \quad \forall \lambda, \mu \in P,
$$

where

$$
\theta_{u, w}(\lambda, \mu)=(\lambda, \mu)_{u}-(w \lambda, w \mu)_{u} .
$$

Proof. Recall that $\sigma(\lambda, \mu)=q^{i \theta_{u, w}(\lambda, \mu)}$ and that card $\hat{P}<\infty$ iff $\sigma(\lambda, \mu)$ is a root of unity for all $\lambda, \mu$.

4.6.5. If $w \neq 1$, then for almost all $u$ a symplectic leaf $\Sigma(1, u) \subset K_{w}$ is equal to $K_{w}$, provided rank $m$ is even, and is dense in $K_{w}$, provided $m$ is odd. If $w=1$ then irreducible representations are one-dimensional and parameterized by points of maximal torus $T$.

Further, if $w \neq 1$, then for almost all $q, u$ we have $P_{Z}=0$ and the centre of $A(P, \sigma)$ is trivial. Hence, for these $w, q, u$ all irreducible representations of $\mathbb{C}\left[K^{u}\right]_{q, w}$ have common kernel.

So, for almost all $u, q$ there are natural bijections "symplectic leaves $\leftrightarrow$ kernels of irreducible representations of $\mathbb{C}\left[K^{u}\right]_{q}$ ", provided $m$ is even, and "closures of symplectic leaves $\leftrightarrow$ kernels of irreducible representations of $\mathbb{C}\left[K^{u}\right]_{q}$, provided $m$ is odd.

4.6.6. Remark. We have no complete theory that can describe the relation between representations of $\mathbb{C}\left[K^{u}\right]_{q}$ and symplectic leaves of a Poisson Lie group $K^{u}$. We think that this study is of interest.

4.6.7. Consider the $C^{*}$-closure of $\mathbb{C}[K]_{q}$ and that of $\mathbb{C}\left[K^{u}\right]_{q}$ with respect to the norm $\|f\|=\sup \|\pi(f)\|$, where $\pi$ is an irreducible representation. We obtain $C^{*}$ algebras $C(K)_{q}^{\pi}$ and $C\left(K^{u}\right)_{q}$ of continuous functions on corresponding quantum groups. To clarify the above consideration we note that

a) (4.7) is necessary and sufficient condition for $C\left(K^{u}\right)$ to be $C^{*}$-algebra of type I (see [23]);

b) $C(K)_{q}$ is always $C^{*}$-algebra of type $\mathrm{I}$.

The spectrum Prim $C(K)_{q}$ is an union $\bigcup_{w \in W} T_{w}$ of tori $T_{w}$ of dimension $m$. The Jackobson topology in Prim $C(K)_{q}$ is consistent with Bruhat order in $W$ :

$$
\bar{T}_{w_{1}} \supset T_{w_{2}} \text { iff } w_{1} \geqq w_{2} .
$$

We do not investigate algebras of continuous functions on compact quantum groups. In [38] general properties of these algebras were studied and in [31, 33] realizations of these algebras and their relatives as algebras of operator-valued functions on torus were obtained. As far as we know there exist no general theorems on such realizations as yet. 
4.7. In this subsection, we study an algebra $\mathbb{C}[K(0, u)]_{q}$. With the notation of 2.7, let $L_{(\lambda, \mu)}$ denote the weight space spanned by $C_{-\lambda, \mu, \Omega}^{\Lambda}$. Clearly,

$$
\mathbb{C}[K(0, u)]_{q}=\underset{(\lambda, \mu) \in P^{2}}{\bigoplus_{(\lambda, \mu)}}
$$

is a graded algebra. The set of maximal two-sided $P^{2}$-graded *-ideals in $\mathbb{C}[K(0, u)]_{q}$ is denoted by $\psi_{q}$.

4.7.1. Remark. By definition, $\mathbb{C}[K(0, u)]_{q}$ and $\mathbb{C}[K]=\mathbb{C}[K(0,0)]_{1}$ coincide as sets. From definition (2.13) of the product in $\mathbb{C}[K(0, u)]_{q}$ it follows that $\psi_{q}=\psi_{1}$ under this identification.

4.7.2. Definition. Let $\pi$ be irreducible $*$-representation, and let $\alpha \in \psi_{q}$ be the maximal element in the set $\left\{\beta \in \psi_{q} \mid \beta \subset \operatorname{Ker} \pi\right\}$. Then we write $\alpha=\operatorname{Gr}(\pi)$.

Set

$$
\begin{gathered}
\Gamma_{\alpha}=\left\{(\lambda, \mu) \in P^{2} \mid L_{(\lambda, \mu)} \not \alpha\right\}, \\
\Gamma(\pi)=\left\{(\lambda, \mu) \in P^{2} \mid L_{(\lambda, \mu)} \not \operatorname{Ker} \pi\right\} .
\end{gathered}
$$

4.7.3. Proposition. a) $\Gamma(\pi)$ is a sublattice in $P^{2}$.

b) $\Gamma(\pi)=\Gamma_{\alpha}$, where $\alpha=\operatorname{Gr}(\pi)$.

Proof. a) From (2.15) and Proposition 2.8, it follows that if $f_{1}, f_{2} \in L_{(\lambda, \mu)}$ then the element $f_{1}^{*} \circ f_{2}=f_{2} \circ f_{1}^{*}$ is central. Let $f \in L_{(\lambda, \mu)}, g \in L_{(\gamma, \delta)}$ satisfy the conditions $\pi(f) \neq 0, \pi(g) \neq 0$; then $f \circ g \in L_{(\lambda+\gamma, \mu+\delta)}$ and

$$
\pi(f \circ g) \pi\left(g^{*} \circ f^{*}\right)=\pi(f) \pi\left(g \circ g^{*}\right) \pi(f)=\pi\left(f \circ f^{*}\right) \pi\left(g \circ g^{*}\right) \neq 0 .
$$

Hence, $(\lambda+\gamma, \mu+\delta) \in \Gamma(\pi)$.

b) Since $\alpha \subset \operatorname{Ker} \pi$, we have $\Gamma(\pi) \subset \Gamma_{\alpha}$. To prove the inclusion $\Gamma_{\alpha} \subset \Gamma(\pi)$, assume that there exists $(\lambda, \mu) \in \Gamma_{\alpha} \backslash \Gamma(\pi)$, choose $f \in L_{(\lambda, \mu)} \backslash \alpha$ such that $\pi(f)=0$, and consider the minimal $\alpha_{1} \in \psi_{q}$, containing both $\alpha$ and $f$. Clearly, $\alpha \subset \alpha_{1} \subset \operatorname{Ker} \pi$, but this contradicts the maximality of $\alpha$.

4.7.4. Proposition. If $\pi: \mathbb{C}[K(0, u)]_{q} \rightarrow$ End $H$ is an irreducible *-representation then $\operatorname{dim} \pi\left(L_{(\lambda, \mu)}\right)=1$ for all $(\lambda, \mu) \in \Gamma(\pi)$.

Proof. $\pi\left(L_{(\lambda, \mu)}\right) \neq\{0\}$ by definition of $\Gamma(\pi)$. Take $f_{1} \circ f_{2} \in L_{(\lambda, \mu)}$ such that $\pi\left(f_{1}\right) \neq 0$, $\pi\left(f_{2}\right) \neq 0$. Since $f_{1} \circ f_{2}^{*}$ and $f_{2}^{*} \circ f_{2}$ are central, we have

$$
\pi\left(f_{1}\right)=\pi\left(f_{1}\right) \pi\left(f_{2}^{*}\right) \pi\left(f_{2}\right) \pi\left(f_{2}^{*} \circ f_{2}\right)^{-1}=d \pi\left(f_{2}\right),
$$

where

$$
d=\pi\left(f_{2}^{*} \circ f_{2}\right)^{-1} \pi\left(f_{1} \circ f_{2}^{*}\right) \in \mathbb{C} .
$$

4.7.5. Proposition. For $(\lambda, \mu) \in \Gamma_{\alpha}=\Gamma(\pi)$ there exist $a_{\lambda, \mu} \in \pi\left(L_{(\lambda, \mu)}\right)$ such that for all $(\lambda, \mu),\left(\lambda^{\prime}, \mu^{\prime}\right) \in \Gamma_{\alpha}$

$$
\begin{gathered}
a_{\lambda, \mu}^{*}=a_{-\lambda,-\mu}=a_{\lambda, \mu}^{-1}, \\
a_{\lambda, \mu} \cdot a_{\lambda^{\prime}, \mu^{\prime}}=\sigma\left((\lambda, \mu),\left(\lambda^{\prime}, \mu^{\prime}\right)\right) a_{\lambda+\lambda^{\prime}, \mu+\mu^{\prime}} .
\end{gathered}
$$

Proof. Choose $a_{\lambda, \mu}$ with the property (4.8) for generators $(\lambda, \mu)$ of $\mathbb{Z}$-module $\Gamma_{\alpha}$ and define $a_{\lambda, \mu}$ for other $(\lambda, \mu) \in \Gamma_{\alpha}$ by formula (4.9). 
4.7.6. Define $\sigma_{\alpha, u, q}: \Gamma_{\alpha} \times \Gamma_{\alpha} \rightarrow \mathbb{C}$ by

$$
\sigma_{\alpha, u, q}\left((\lambda, \mu),\left(\lambda^{\prime}, \mu^{\prime}\right)\right)=q^{i\left[-\left(\lambda, \lambda^{\prime}\right)_{u}+\left(\mu, \mu^{\prime}\right)_{u}\right]}
$$

and construct quantum torus $A\left(\Gamma_{\alpha}, \sigma_{\alpha, u, q}\right)$.

4.7.7. Choose $a_{\lambda, \mu}$ for $(\lambda, \mu) \in \pi\left(L_{(\lambda, \mu)}\right)$ as in Proposition 4.7.5, set for $f \in L_{(\lambda, \mu)}$ and $U_{\lambda, \mu} \in A\left(\Gamma_{\alpha}, \sigma_{\alpha, u, q}\right) \pi_{0}(f)=\pi(f) a_{\lambda, \mu}^{-1} \in \mathbb{C}, \quad \chi\left(U_{\lambda, u}\right)=a_{\lambda, u}\left((\lambda, \mu) \in \Gamma_{\alpha}\right), \quad \pi_{0}\left(L_{(\lambda, \mu)}\right)=\{0\}$, $(\lambda, \mu) \notin \Gamma_{\alpha}$, and extend $\pi_{0}, \chi$ by linearity to the maps

$$
\pi_{0}: \mathbb{C}[K] \rightarrow \mathbb{C}, \quad \chi: A\left(\Gamma_{\alpha}, \sigma_{\alpha, u, q}\right) \rightarrow \text { End } H .
$$

4.7.8. Theorem. $\pi_{0}$ and $\chi$ are irreducible $*$-representations.

Proof. From formulae (4.8), (4.9) and definition of the product in $\mathbb{C}[K(0, u)]_{q}$, it follows that $\pi_{0}$ and $\chi$ are $*$-representations. Clearly, $\pi_{0}$ is irreducible and $\chi$ is irreducible since $\pi$ is.

4.7.9. Theorem. Let $\pi_{0}: \mathbb{C}[K] \rightarrow \mathbb{C}$ be an irreducible *-representation, let $\alpha=\operatorname{Gr}\left(\pi_{0}\right)$, and let $\chi: A\left(\Gamma_{\alpha}, \sigma_{\alpha, u, q}\right) \rightarrow$ End $H$ be an irreducible *-representation. Then the map $\pi: \mathbb{C}[K(0, u)]_{q} \rightarrow$ End $H$, defined by

$$
\pi(f)= \begin{cases}\pi_{0}(f) \chi\left(U_{\lambda, \mu}\right), & f \in L_{(\lambda, \mu)}, \\ 0, \quad f \in L_{(\lambda, \mu)}, & (\lambda, \mu) \notin \Gamma_{\alpha},\end{cases}
$$

and extended to $\mathbb{C}[K(0, u)]_{q}$ by linearity, is an irreducible *-representation.

Proof. By definition of the products in $\mathbb{C}[K(0, u)]_{q}$ and $A\left(\Gamma_{\alpha}, \sigma_{\alpha, u, q}\right)$, the map $\pi$ is *-representation. Its irreducibility is evident.

4.7.10. Definition. Pairs $\left(\pi_{0}, \chi\right)$ and $\left(\pi_{0}^{\prime}, \chi^{\prime}\right)$ are said to be equivalent provided $\operatorname{Gr}\left(\pi_{0}\right)$ $=\operatorname{Gr}\left(\pi_{0}^{\prime}\right)$ and there exists linear real-valued function $\varphi$ on $\Gamma_{\alpha}$ such that

$$
\pi_{0}^{\prime}(f)=e^{-i \varphi(\lambda, \mu)} \pi_{0}(f), \quad \chi^{\prime}\left(U_{\lambda, \mu}\right)=e^{i \varphi(\lambda, \mu)} \chi\left(U_{\lambda, \mu}\right)
$$

for all $(\lambda, \mu) \in \Gamma_{\alpha}$ and all $f \in L_{(\lambda, \mu)}$.

The following statement is evident.

4.7.11. Proposition. Pairs $\left(\pi_{0}, \chi\right)$ and $\left(\pi_{0}^{\prime}, \chi^{\prime}\right)$ determine the same representation $\pi$ iff they are equivalent.

Thus, irreducible $*$-representations of $\mathbb{C}[K(0, u)]_{q}$ are parametrized by pairs $\left(\pi_{0}, \chi\right)$ up to the equivalence of pairs. Here $\pi_{0}$ is an irreducible $*$-representation of $\mathbb{C}[K]$ and $\chi$ is an irreducible *-representation of the quantum torus $A\left(\Gamma_{\alpha}, \sigma_{\alpha, u, q}\right)$, where $\alpha=\operatorname{Gr}\left(\pi_{0}\right)$.

\section{Quantum Weyl Group}

5.1. In this section we'll give the application of results of Sect. 3 to the definition of the quantum Weyl group. The proofs given in our article [14] will be omitted.

The connection between the quantum Weyl group, Lusztig's [16] automorphisms, and the formula for the universal $R$-matrix is described. Similar results were independently obtained in [8]. 
5.2. In Subsect. 3.7, for each simple reflection $s_{i} \in W$ its quantum analog $\bar{s}_{i} \in \mathbb{C}[K]_{q}^{V}$ was defined. From Proposition 3.7.1, it follows that for every $w \in W$ its quantum analog $\bar{w}$ is well-defined. For a reduced expression $w=s_{i_{1}} \ldots s_{i_{\mathrm{K}}}$ the element $\bar{w}=\bar{s}_{i_{1}} \ldots \bar{s}_{i_{K}}$ is GNS-state corresponding to the representation $\pi_{w}$ and the highest vector of this representation.

In this section it is convenient to deal with the algebra $\mathbb{C}[K]_{h}$ instead of $\mathbb{C}[K]_{q}$ (recall that $q=\exp (h / 2)$ and that $\mathbb{C}[K]_{h}$ is an algebra over the ring $\mathbb{C} \llbracket h \rrbracket$ of formal series in $h$ ). From considerations of Subsect. 2.7 it follows that we still can use the results on $\mathbb{C}[K]_{q}$.

5.3. In $U_{k}(g)$, define the following elements:

$$
E_{i}=X_{i}^{+} q^{-\frac{H_{i}}{2}}, \quad F_{i}=X_{i}^{-} q^{\frac{H_{i}}{2}}, \quad e_{i}=q^{\frac{H_{i}}{2}} X_{i}^{+}, \quad f_{i}=q^{-\frac{H_{i}}{2}} X_{i}^{-}
$$

and, for $t>1$ and $n \in \mathbb{N}$, set

$$
(n)_{t}=\frac{t^{n}-1}{t-1}, \quad(n)_{t} !=(1)_{t} \ldots(n)_{t}, \quad \exp _{t}(x)=\sum_{n \geqq 0} \frac{x^{n}}{(n)_{t} !},
$$

where $(0)_{t}=1$.

5.3.1. Proposition [31]. In $\mathbb{C}[K]_{h}^{V}$, the following relations hold:

a) $\bar{s}_{i} E_{i} \bar{s}_{i}^{-1}=-q^{-H_{i}} F_{i}$,

b) $\bar{s}_{i} F_{i} \bar{s}_{i}^{-1}=-E_{i} q^{H_{i}}$.

Set

$$
\begin{gathered}
q_{i}=q^{\left(\alpha_{i}, \alpha_{i}\right) / 2}, \\
\widetilde{R}_{i}=\exp _{q_{i}-2} 2\left(\left(1-q_{i}^{-2}\right) e_{i} \otimes f_{i}\right)=\sum_{n \geqq 0} \frac{\left(1-q_{i}^{-2}\right)^{2}}{(n)_{q_{i}-2} !} e_{i}^{n} \otimes f_{i}^{n}, \\
R_{i}=\left(\sum_{n \geqq 0} \frac{\left(1-q_{i}^{-2}\right)^{n}}{(n)_{q_{i}-2} !} E_{i}^{n} \otimes F_{i}^{n}\right) q^{\frac{H_{i} \otimes H_{i}}{\left(\alpha_{i}, \alpha_{i}\right)}} \\
=q^{\frac{H_{i} \otimes H_{i}}{\left(\alpha_{i}, \alpha_{i}\right)}} \sum_{n>0} \frac{\left(1-q_{i}^{-2}\right)^{n}}{(n)_{q_{i}-2} !} e_{i}^{n} \otimes f_{i}^{n}, \\
\bar{s}_{i}^{\prime}=q^{-\frac{H_{i}^{2}}{2\left(\alpha_{i}, \alpha_{i}\right)} \bar{s}_{i} .}
\end{gathered}
$$

5.3.2. Proposition $[9,14,28,31]$. Let $\Delta, \varepsilon$, and $S$ be the comultiplication, the counit and the antipode in $\mathbb{C}[K]_{h}^{V}$. Then
a) $\Delta\left(\bar{s}_{i}^{\prime}\right)=R_{i}^{-1}\left(\bar{s}_{i}^{\prime} \otimes \bar{s}_{i}^{\prime}\right), \quad \Delta\left(\bar{s}_{i}\right)=\exp _{q_{i}^{-2}}\left(\left(1-q_{i}^{-2}\right) e_{i} \otimes f_{i}\right)$,
b) $\varepsilon\left(\bar{s}_{i}^{\prime}\right)=\varepsilon\left(\bar{s}_{i}\right)=1$,
c) $S\left(\bar{s}_{i}^{\prime}\right)=\left(\bar{s}_{i}^{\prime}\right)^{-1} \sum_{n \geqq 0} \frac{\left(1-q_{i}^{-2}\right)^{n}}{(n)_{q_{i}-2} !} E_{i}^{n} q^{-\frac{H_{i}^{2}}{\left(\alpha_{i}, \alpha_{i}\right)}} S\left(F_{i}^{n}\right)$.

The next proposition is the substitute for the relation $\bar{s}_{i}^{2}=1$.

5.3.3. Proposition $[14,28] .\left(\bar{s}_{i}^{\prime}\right)^{2}=e^{-h C_{i} / 2}$, where $e^{-h C_{i} / 2}$ is the quantum analog, defined in Sect. 4 of [4], for the Casimir element.

Recall that in a Hopf algebra $A$ two adjoint actions are defined: if $\Delta(a)$ $=\sum_{i} a_{i}^{1} \otimes a_{i}^{2}$, then

$$
\operatorname{ad}_{a}(x)=\sum_{i} a_{i}^{1} x S\left(a_{i}^{2}\right), \quad \operatorname{ad}_{a}^{\prime}(x)=\sum_{i} a_{i}^{2} x S\left(a_{i}^{1}\right) .
$$


5.3.4. Proposition [14].For $i \neq j$, the following relations hold:

a) $\bar{s}_{i} E_{j} \bar{s}_{i}^{-1}=C_{i j}^{+}(h) \operatorname{ad}_{E_{i}}^{-a_{i j}}\left(E_{j}\right)$,

b) $\bar{s}_{i} F_{j} \bar{s}_{i}^{-1}=C_{i j}^{-}(h)\left(\operatorname{ad}_{F_{i}}^{\prime}\right)^{-a_{i j}}\left(F_{j}\right)$,

where $\left(\left(a_{i j}\right)\right)$ is the Cartan matrix of $\mathscr{Y}$, and $C_{i j}^{ \pm}(h)$ are as follows:

$$
\begin{gathered}
C_{i j}^{ \pm}(h)=\sqrt{(-1)^{a_{i j}} \prod_{K=0}^{\left(-a_{i j}-1\right)}\left(\sum_{m=0}^{K}\left[\left(\alpha_{i}, \alpha_{j}\right)+m\left(\alpha_{i}, \alpha_{i}\right)\right]_{q}\right)}, \\
{[n]_{q}=\frac{q^{n}-q^{-n}}{q-q^{-1}}, \quad \varepsilon_{i j}^{ \pm}=\left\{\begin{array}{ll}
0 & \text { for }+ \\
1 & \text { for }-
\end{array} .\right.}
\end{gathered}
$$

5.3.5. Corollary [14]. Set $T_{i}(x)=\operatorname{Ad}_{\bar{s}_{i}}(x)=\bar{s}_{i}, x \bar{s}_{i}^{-1}$. Then $T_{i}$ coincide up to normalization with Lusztig's automorphisms [16].

5.4. Definition. The minimal Hopf subalgebra in $\mathbb{C}[K]_{h}^{V}$ containing $U_{h}(g)$ and GNS-states $\left\{\bar{s}_{i}\right\}$, is called the quantum Weyl group and is denoted by $\bar{U}_{h}(g)$.

5.4.1. Remark. a) From 5.3.1-5.3.4, it follows that $\overparen{U_{h}(g)}$ is well-defined and that $\bar{s}_{i}^{-1} \in \overline{U_{h}(g)}$.

b) From 5.3.2a), c), it is easy to see the impossibility of construction of a Hopf algebra containing elements $\bar{s}_{i}$ only. Here is the distinction between the quantum case and the classical one: in the latter case $\Delta\left(s_{i}\right)=s_{i} \otimes s_{i}$.

5.5. Let $w_{0}$ be the longest element in the Weyl group. Fix a reduced expression $w_{0}$ $=s_{i_{1}} \ldots s_{i_{N}}$ and define the quantum analogs of root vectors as follows $[14,16]$ :

a) $E_{\alpha_{i_{1}}}=E_{i_{1}}, F_{\alpha_{i_{1}}}=F_{i_{1}}$,

b) If $\alpha=s_{i_{1}} \ldots \chi_{i_{P-1}} \alpha_{i_{P}}$ then $E_{\alpha}=T_{w_{P}}\left(E_{i_{P}}\right), F_{\alpha}=T_{w_{P}}\left(F_{i_{P}}\right)$, where $T_{w_{P}}=T_{i_{1}} \ldots T_{i_{P-1}}$. This is possible as $\Delta_{+}=\left\{\alpha_{i_{1}}, s_{i_{1}} \alpha_{i_{2}}, \ldots, s_{i_{1}} \ldots s_{i_{N-1}} \alpha_{i_{N}}\right\}$.

Set $\alpha(1)=\alpha_{i_{1}}, \alpha(2)=S_{i_{1}} \alpha_{i_{2}}, \ldots$ and introduce in $\Delta_{+}$the order $\alpha(1)<\alpha(2)$ $<\ldots<\alpha(N)$.

Let $U_{h}\left(n_{+}\right), U_{h}\left(n_{-}\right), U_{h}(\mathfrak{h}), U_{h}\left(b_{+}\right), U_{h}\left(b_{-}\right)$denote the unital subalgebras generated by $\left\{E_{\alpha}\right\} ;\left\{F_{\alpha}\right\} ;\left\{H_{i}\right\} ;\left\{E_{\alpha}, H_{i}\right\} ;\left\{F_{\alpha}, H_{i}\right\}$ respectively.

5.5.1. Theorem $[14,16]$. The monomials

$$
\left\{E_{\alpha(1)}^{K_{1}} \ldots E_{\alpha(N)}^{K_{N}} H_{1}^{\ell_{1}} \ldots H_{m}^{\ell_{m}}\right\}_{\ell_{i}, K_{i} \in \mathbb{Z}_{+}}
$$

form a linear basis in $U_{h}\left(b_{+}\right)$and the monomials

$$
\left\{E_{\alpha(1)}^{K_{1}} \ldots E_{\alpha(N)}^{K_{N}} H_{1}^{\ell_{1}} \ldots H_{m}^{\ell_{m}} F_{\alpha(1)}^{P_{1}} \ldots F_{\alpha(N)}^{P_{N}}\right\}_{K_{i}, \ell_{i}, P_{i} \in \mathbb{Z}_{+}}
$$

form a linear basis in $U_{h}(g)$.

5.5.2. Proposition [14]. If $\alpha<\beta$ then

$$
E_{\alpha} E_{\beta}-q^{-(\alpha, \beta)} E_{\beta} E_{\alpha}=\sum_{\alpha<\gamma_{1}<\ldots<\gamma_{j}<\beta} C_{\bar{K}, \bar{\gamma}} E_{\gamma_{1}}^{K_{1}} \ldots E_{\gamma_{j}}^{K_{j}}
$$

and a similar formula holds for $F_{\alpha}$ 's.

Proof. From the P.B.W. Theorem 5.5.1 it follows that for small $h$

$$
E_{\beta} E_{\alpha}=\sum_{\bar{m}} C_{\bar{m}} E_{\alpha(1)}^{m_{1}} \ldots E_{\alpha(N)}^{m_{N}} .
$$


Let $\alpha=\alpha(\ell)<\beta=\alpha(K)$, i.e. $\ell<K$. We show first that in the right side of (5.2) there are no summands with $C_{m_{S}} \neq 0$ and $m_{S}>0$ for $S>K$. The proof is based on the following

5.5.3. Lemma. Let $w \in W$. If $w^{-1} \alpha_{i}>0$, then $T_{w}^{-1}\left(E_{i}\right) \in U_{h}\left(n_{+}\right)$, and if $w^{-1} \alpha_{i}<0$ then $T_{w}^{-1}\left(E_{i}\right) \in U_{h}\left(n_{-}\right)_{t}$, where $t \in U_{h}(\mathfrak{h})$.

The analogous statements hold after substitution of $F_{i}$ for $E_{i}$ and - for + .

The proof is essentially the same as that of the analogous result of [18], the following formulae being used

$$
\begin{aligned}
& T_{i}^{-1}\left(E_{j}\right)=C_{i j}^{ \pm}(h) q_{i}^{-a_{i j}}\left(-\operatorname{ad}_{q^{-1}, E_{i}}\right)^{-a_{i j}}\left(E_{j}\right), \\
& T_{i}^{-1}\left(F_{j}\right)=C_{i j}^{-}(h) q_{i}^{-a_{i j}}\left(-\operatorname{ad}_{q^{-1}, F_{i}}^{\prime}\right)^{-a_{i j}}\left(F_{j}\right) .
\end{aligned}
$$

Here $i \neq j, C_{i j}^{ \pm}(h)$ are defined in 5.3.4 and $\mathrm{ad}_{q^{-1}}$ denotes the adjoint action in the Hopf algebra $U_{-h}(g)$.

5.5.4. We return to proof of Proposition 5.5.2. Assume that in the right side of (5.2) there is a monomial $E_{\alpha(1)}^{m_{1}} \ldots E_{\alpha(P)}^{m_{P}}$ with $m_{P}>0, P>K$. We can assume $P$ being the maximal number with this property. Set $w_{P}=s_{i_{1}} \ldots s_{i_{P-1}}$ and apply $T_{w_{P}}^{-1}$ to both sides of $(5.2)$. Since $w_{P}^{-1}(\alpha(P))>0$ and $w_{P}^{-1}(\alpha(s))<0$, it follows from Lemma 5.5.3, that on the left in (5.2) we get an element from $U_{h}\left(b_{-}\right)$. Moreover, all the summands on the right side in (5.2), except one, belong to $U_{h}\left(b_{-}\right)$as well, and this last summand is of the form $\xi E_{\alpha(P)}^{m_{P}}$, where $\xi \in U_{h}\left(b_{-}\right)$. By P.B.W. theorem, this contradicts the assumption $m_{P}>0$ (for small $h$ ).

Thus in (5.2) $m_{P}=0$ for $P>K$ and in the same way one can show that $m_{P}=0$ for $P<K$, too [one assumes that $P$ is the minimal number with the property $m_{P}>0$ and applies $T_{w_{P+1}}^{-1}$ to both sides of (5.2)].

Thus, for some $a \in \mathbb{C}$,

$$
E_{\beta} E_{\alpha}-a E_{\alpha} E_{\beta}=\sum_{\alpha<\gamma_{1}<\ldots<\gamma_{j}<\beta} C_{\bar{m}, \bar{\gamma}} E_{\gamma_{1}}^{m_{1}} \ldots E_{\gamma_{j}}^{m_{j}} .
$$

We apply $T_{w_{t+1}}^{-1}$ to both sides and obtain

$$
T_{i_{\ell}} \ldots T_{i_{K-1}}\left(E_{i_{K}}\right)\left(F_{i_{\ell}} q^{H_{i_{\ell}}}\right)-a\left(F_{i_{\ell}} q^{H_{i}}\right) T_{i_{\ell}} \ldots T_{i_{K-1}}\left(E_{i_{K}}\right) \in U_{h}\left(b_{-}\right)
$$

(see Lemma 5.5.3 and Proposition 5.3.1). By P.B.W. theorem, from this follows that $a=q^{-(\alpha, \beta)}$ as needed for (5.1).

5.6. In this subsection we apply above results to the calculation of universal $R$-matrices for quantized universal enveloping algebras $U_{h, u}(g)$ from Sect. 2. Note that for $u \neq 0$ the corresponding $R$-matrix is of the form $R_{u}=e^{h u / 2} R e^{h u / 2}$, where $R$ is the universal $R$-matrix for $U_{h}(g)$, defined in Sect. 13 of [3]. Recall that the universal $R$-matrix is an invertible element of $U_{h}(g) \otimes U_{h}(g)$ which satisfies the equations

$$
\begin{gathered}
\Delta^{\prime}(a)=R \Delta(a) R^{-1}, \\
(\Delta \otimes \mathrm{id})(R)=R^{13} R^{23}, \quad(\operatorname{id} \otimes \Delta)(R)=R^{12} R^{13}
\end{gathered}
$$

and has the form of the special series (for details, see $[3,4]$ ). Here $\Delta^{\prime}$ is the opposite comultiplication, $R^{12}=R \otimes 1, \quad R^{23}=1 \otimes R, R^{13}=(1 \otimes \sigma)\left(R^{12}\right)$, where $\sigma(a \otimes b)$ $=b \otimes a$. In Sect. 13 of [3], there is a receipt for construction of the universal 
$R$-matrix. First, one constructs the Hopf algebra $\mathscr{D}\left(U_{h}\left(b_{+}\right)\right)$, i.e. the so-called quantum double of Hopf algebra $U_{h}\left(b_{+}\right)$. Note that $\mathscr{D}\left(U_{h}\left(b_{+}\right)\right) \cong U_{h}\left(b_{+}\right) \otimes U_{h}\left(b_{+}\right)^{V}$ as vector spaces and there is a natural Hopf algebra isomorphism $U_{h}\left(b_{+}\right)^{0}$ $\cong U_{h}\left(b_{-}\right)$, where $U_{h}\left(b_{+}\right)^{0}$ is the Hopf algebra $U_{h}\left(b_{+}\right)^{V}$ with the opposite comultiplication. From this, one can derive that $R$ is the image under projection

$$
\pi: \mathscr{D}\left(U_{h}\left(b_{+}\right)\right) \otimes \mathscr{D}\left(U_{h}\left(b_{+}\right)\right) \rightarrow U_{h}(g) \otimes U_{h}(g)
$$

of the canonical element $\bar{R}=\sum_{K} e_{K} \otimes e^{K}$ where $\left\{e_{K}\right\}$ is a linear basis in $U_{h}\left(b_{+}\right)$, and $\left\{e_{K}\right\}$ is the dual basis in $U_{h}\left(b_{+}\right)^{\frac{K}{V}}$. Using the results of Subsect. 5.5, we can construct these bases, and using 5.3.2 and 5.5.2 we can explicitly describe the isomorphism $U_{h}\left(b_{+}\right)^{0} \cong U_{h}\left(b_{-}\right)$.

Finally, we come to the following

5.6.1. Proposition [8, 14]. The following formula for the universal R-matrix in $U_{h}(g)$ holds:

$$
R=\sum_{\alpha \in \Delta_{+}}^{g} \exp _{q_{\bar{\alpha}} 2}\left(\left(1-q_{\alpha}^{-2}\right) E_{\alpha} \otimes F_{\alpha}\right) q^{t_{0}} .
$$

Here $q_{\alpha}=q^{\frac{(\alpha, \alpha)}{2}}=e^{\frac{h(\alpha, \alpha)}{4}}, t_{0} \in \mathfrak{h} \otimes \mathfrak{h}$ is the canonical element corresponding to inner product in $\mathfrak{h}, E_{\alpha}$ and $F_{\alpha}$ are defined in 5.5, and the product is taken in the order opposite to that in 5.5 .

5.6.2. Remark. a) In [15-18] there are many interesting applications of the quantum Weyl group. All of them are related to automorphisms $T_{i}$ corresponding to $\bar{s}_{i}$ (see 5.3.5), but not to the latter. We believe that the existence of the Hopf algebra $\overline{U_{h}(g)}$ from 5.4 is of interest per se. Sometimes the Hopf algebra structure in $\overline{U_{h}(g)}$ allows to simplify the proofs of the results related to $T_{i}$ (see [14, Sect. 4]). In the case of Proposition 5.6.1, the knowledge of the Hopf algebra structure in $\overline{U_{h}(g)}$ is necessary to obtain the result. We believe that the axiomatic description of quantum groups (i.e. Hopf algebras with invertible antipode) "having Weyl group" is of interest.

b) Note the following important formula, which follows from 5.6.1. Let $\left\{I_{K}\right\}$ be the orthonormal basis in $\mathfrak{h}$ and $\bar{w}_{0}=\bar{s}_{i_{1}} \ldots \bar{s}_{i_{N}}$ be the quantum analog of the longest element in Weyl group. Set

$$
\bar{w}_{0}^{\prime}=q^{-\frac{1}{2} \sum_{K} I_{K}} \bar{w}_{0} .
$$

Then $[8,14,28]$

$$
\Delta\left(\bar{w}_{0}^{\prime}\right)=R^{-1}\left(\bar{w}_{0}^{\prime} \otimes \bar{w}_{0}^{\prime}\right) .
$$

Using (5.4), one can show that $\left(\bar{w}_{0}^{\prime}\right)^{2}=e^{-h C / 2}$, where $e^{-h C / 2}$ is the quantum analog of Casimir element from [4].

Acknowledgements. We wish to express gratitude to V. Drinfeld for valuable advices. We also thank L. Faddeev, A.N. Kirillov, N. Reshetikhin, M. Semenov-Tian-Shansky, and L. Vaksman for useful discussions. 


\section{References}

1. Belavin, A., Drinfeld, V.: The triangle equations and simple Lie algebras. Preprint of Inst. Theor. Phys., No. 18 (1982)

2. Dixmier, J.: Les $C^{*}$-algèbres et leurs representations. Paris: Gauthier-Villars éditeur 1969

3. Drinfeld, V.: Quantum groups. In: Proc. ICM, Berkeley, 1986, Providence, RI: Am. Math. Soc., 1988, pp. 798-820

4. Drinfeld, V.: On almost cocommutative Hopf algebras. Algebra and Analiz. 1(2), 30-49 (1989)

5. Faddeev, L.D., Reshetikhin, N.Yu., Takhtajan, L.A.: Quantization of Lie groups and Lie algebras. In: Algebraic analysis, Vol. 1, 129-139. New York: Academic Press 1988

6. Jimbo, M.: Quantum R-matrix for GTS: algebraic approach. Lecture Notes in Physics, Vol. 246. Berlin, Heidelberg, New York: Springer 1986

7. Kirillov, A.: The elements of representation theory. Moskow: Nauka 1972

8. Kirillov, A.N., Reshetikhin, N.Yu.: $Q$-analog of Weyl group and $R$-matrices. Preprint (1990) (to appear)

9. Kirillov, A.N., Reshetikhin, N.Yu.: Quantum group $S U(2)$, $q$-orthogonal polynomials and links invariants. Preprint LOMI (1988)

10. Koelink, H.: On *-representations of the Hopf $*$-algebra associated with the quantum group $U_{q}(n)$. Preprint Math. Inst. Univ. of Leiden (1989)

11. Koornwinder, T.H.: Representations of the twisted $S U(2)$ quantum group and some $q$-hypergeometric orthogonal polynomials. Proc. Koninklijke Nederlandse Akad. Wetenschappen, Series A 92, 97-117 (1989)

12. Koornwinder, T.H.: Orthogonal polynomials in connection with quantum groups. In: Orthogonal polynomials: theory and practice. NATO ASI Series C, Vol. 294, pp. 257-292. London: Kluwer 1989

13. Levendorskiǐ, S.: Twisted algebras of functions on compact quantum groups and their representations. Algebra Analiz (to appear)

14. Levendorskiǐ, S., Soibelman, Ya.: Some applications of quantum Weyl group, J. Geom. Phys. 7 (4), 1-14 (1991)

15. Lusztig, G.: Quantum deformations of certain simple modules over enveloping algebras. Adv. Math. 70, 237-249 (1988)

16. Lusztig, G.: Quantum groups at roots of 1. Preprint, MIT (1989)

17. Lusztig, G.: On quantum groups. J. Algebra (to appear)

18. Lusztig, G.: Finite-dimensional Hopf algebras arising from quantum groups. J. Am. Math. Soc. 3, 257-296 (1990)

19. Majid, Sh.: Quasitriangular Hopf algebras and Yang-Baxter equations. Interl. J. Mod. Phys. A 5(1), 1-91 (1990)

20. Manin, Yu.I.: Quantum groups and non-commutative geometry. Montreal: Centre de Rech. Math. 1988

21. Masuda, T., Mimachi, K., Nakagami, Y., Noumi, M., Ueno, K.: Harmonic analysis on quantum groups and $q$-analog of spherical functions. C.R. Acad. Sci. Paris Sér. 1 Math 307, 559-564 (1988)

22. Reshetikhin, N., Semenov-Tian-Shansky, M.: Quantum $R$-matrices and factorization problems in quantum groups, J. Geom. Phys. (1989)

23. Rieffel, M.A.: Non-commutative tori - a case study of non-commutative differentiable manifolds. Preprint 1989

24. Semenov-Tian-Shansky, M.: Dressing transformations and Poisson Lie group actions. Publ. RIMS. 21, 1237-1260 (1985)

25. Serre, J.-P.: Lie algebras and Lie groups. New York, Amsterdam: Benjamin 1965

26. Soibelman, Ya., Vaksman, L.: Algebra of funetions on the quantized group $S U(2)$, 1. Preprint 1986 (in Russian)

27. Soibelman, Ya., Vaksman, L.: Algebra of functions on the quantized group $S U(2)$, 2. Preprint 1986 (in Russian)

28. Soibelman, Ya.: Algebra of functions on the quantum group $S U(n)$ and Schubert cells. Dokl. AN SSSR 307(1), 41-45 (1989)

29. Soibelman, Ya.: Algebra of functions on the quantum compact groups and its representations. Algebra Analiz 2(1), 190-221 (1990) 
30. Soibelman, Ya.: Gelfand-Naimark-Segal states and Weyl group for quantum group $S U(n)$. Funct. Anal. i ego pril. 24(3) (1990)

31. Soibelman, Ya., Vaksman, L.: Algebra of functions on quantum group $S U(2)$. Funct. Anal. i ego pril. 22(3), 1-14 (1988)

32. Soibelman, Ya., Vaksman, L.: Some questions in quantum group theory. Adv. Sov. Math. (to appear)

33. Soibelman, Ya., Vaksman, L.: Algebra of functions on quantum group $S U(n)$ and odddimensional spheres. Preprint 1989, to appear in Algebra Analiz 2(5) (1990) (in Russian)

34. Steinberg, R.: Lectures on Chevalley groups. Yale Univ. 1972

35. Weinstein, A.: The local structure of Poisson manifolds. J. Diff. Geom. 18, 523-557 (1983)

36. Weinstein, A., Jiang-Hua Lu: Poisson Lie groups, dressing transformations and Bruhat decompositions. J. Diff. Geom. 31, 501-526 (1990)

37. Woronowicz, S.: Twisted SU(2) group. Publ. RIMS. 23(1), 117-181 (1987)

38. Woronowicz, S.: Compact matrix pseudogroup. Commun. Math. Phys. 111, 613-665 (1987)

Communicated by N. Yu. Reshetikhin 